\title{
Status, Challenges and Directions in Indirect Dark Matter Searches
}

\author{
Carlos Pérez de los Heros $(\mathbb{D}$ \\ Department of Physics and Astronomy, Uppsala University, 75120 Uppsala, Sweden; cph@physics.uu.se
}

Received: 20 August 2020; Accepted: 23 September 2020; Published: 8 October 2020

\begin{abstract}
Indirect searches for dark matter are based on detecting an anomalous flux of photons, neutrinos or cosmic-rays produced in annihilations or decays of dark matter candidates gravitationally accumulated in heavy cosmological objects, like galaxies, the Sun or the Earth. Additionally, evidence for dark matter that can also be understood as indirect can be obtained from early universe probes, like fluctuations of the cosmic microwave background temperature, the primordial abundance of light elements or the Hydrogen 21-cm line. The techniques needed to detect these different signatures require very different types of detectors: Air shower arrays, gamma- and X-ray telescopes, neutrino telescopes, radio telescopes or particle detectors in balloons or satellites. While many of these detectors were not originally intended to search for dark matter, they have proven to be unique complementary tools for direct search efforts. In this review we summarize the current status of indirect searches for dark matter, mentioning also the challenges and limitations that these techniques encounter.
\end{abstract}

Keywords: dark matter; indirect detection; WIMP; gamma rays; cosmic rays; neutrinos

\section{Introduction}

The term "dark matter" was originally coined because of the need to explain the observed rotation curves of stars in galaxies and the peculiar velocities of galaxies within clusters. The velocities of these large gravitational systems were measured to be incompatible with the expectations based on Newtonian mechanics and the visible amount of matter in the system [1-3]. These observations seemed to provide indirect evidence for the existence of either a non-luminous matter component in the universe, or a deviation from the standard Newtonian mechanics as we use it to try to make sense of the universe at larges scales [4]. Additional matter is also needed to understand how the first galaxies could have formed from the small density perturbations imprinted in the cosmic microwave background $(\mathrm{CMB})[5]$ and to describe the large-scale structure of the universe as seen today [6,7]. Observations of gravitational lensing added to the list of evidence for the need of dark matter [8]. While proposed modifications of gravity and other recent approaches [9-12] do not need dark matter at all, in this review we summarize the current experimental efforts aimed at probing indirectly the most popular "solution" to the dark matter problem: The particle solution.

The particle solution to the dark matter problem is based on the assumption that stable (or enough long-lived) relic particles exist, whose present density is determined by the thermal history of the early universe [13]. Incidentally, models needed to explain certain shortcomings of the Standard Model of particle physics do provide viable candidates for dark matter in the form of new weakly-interacting massive particles (WIMPs) in the GeV-O $(100 \mathrm{TeV})$ mass range. If they are thermally produced in the early universe, the upper mass limit for WIMPs arises from theoretical arguments in order to preserve unitarity [14]. There is practically no lower limit on the mass of the dark matter candidates as long as they can perform their role of being abundant enough to solve the problem and they do not over close the universe [15]. Higher masses than $O(100 \mathrm{TeV})$ and/or stronger interactions can be accommodated in models where the dark matter candidates are not produced thermally [16]. So the 
dark matter zoo encompasses a wide class of particles with a wide range of interactions as illustrated in Figure 1. From a theoretical point of view, the connection between a cosmological problem (the need for additional matter at cosmological scales) and the possibility of explaining it with particle physics ideas is extremely compelling.

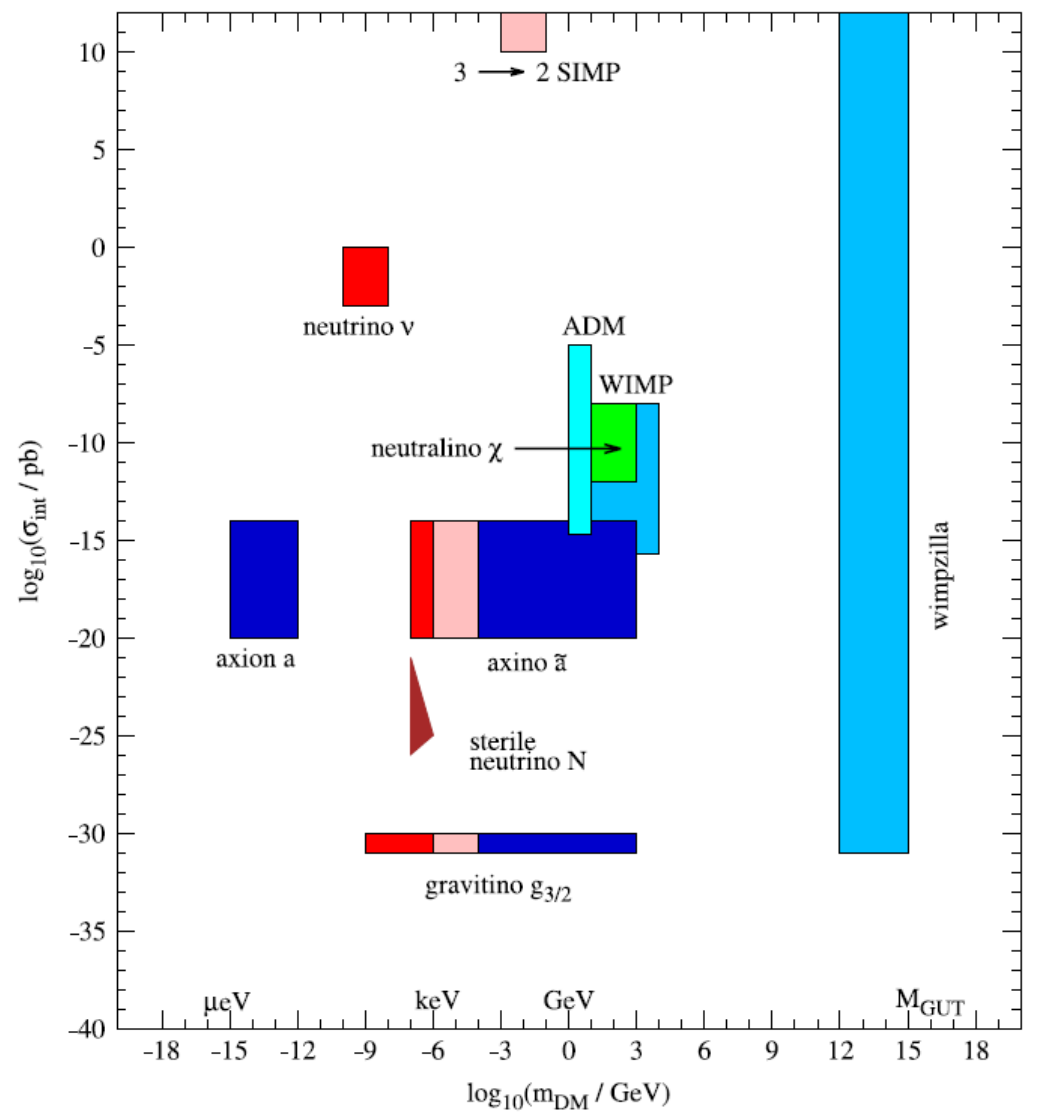

Figure 1. Popular candidates of dark matter indicating their mass and strength of interaction with ordinary matter. The red, pink and blue colors represent hot dark matter (HDM), warm dark matter (WDM) and cold dark matter (CDM) candidates, respectively. Reprinted from [16] with permission from Elsevier.

The term "indirect" appertaining to dark matter searches refers to those methods based on detecting the products of the annihilation or decays of dark matter candidates gravitationally accumulated in cosmological objects, in contrast with "direct" detection techniques where the interactions of dark matter particles from the local halo with a suitable target are searched for [17]. Still, these two techniques rely on detecting the dark matter that surrounds us. Usually not included in "indirect" searches is the use of early universe probes (cosmic microwave background power spectrum, 21-cm astronomy and nucleosynthesis) to set constraints to the dark matter content and characteristics. There is no more indirect way of searching for something that using signatures that might have occurred billions of years ago, so we have included a short description of dark matter searches using those probes at the end of this review. Additionally, accelerator searches try to produce new particles in the controlled conditions of collider or fixed target interactions, that could be identified also as candidates for dark matter [18-20]. In any case, the problem remains to identify and characterize this new form of matter, if this is indeed the way Nature chose to function.

Any review about dark matter, be it about direct or indirect searches, faces at least two, I think insurmountable, problems: To be sufficiently up to date in a rapidly evolving field, and to give due credit to previous work by the innumerable scientists that have contributed to the field. I confess already here that I have given up in trying to solve these two problems. Instead, I have tried to provide 
a personal choice of topics and mention some general problems faced in the field of indirect dark matter searches that I think are relevant and that sometimes can have a bearing on the interpretation of results (the choice of halo model or the consideration of particle physics or astrophysical uncertainties for example). This means that I have not followed a historical approach (there are excellent reviews from that perspective [21,22]), neither I have tried to be complete in describing the theoretical models being usually, or less usually, considered (there are also excellent reviews on that [23-25]), or tried to be comprehensive in mentioning the results of every single experiment that exists in the field. I have also favoured citing published results than preprints or conference proceedings when possible, sometimes at the expense of not pointing the reader to the very latest results. With these caveats in mind, I hope this review is of use to some readers.

\section{Dark Matter in Galactic Halos}

A viable particle candidate for dark matter must be stable in timescales comparable with the age of the universe and have interactions with ordinary matter such it does not affect the evolution of the universe in a way that leads to a large-scale structure that is incompatible with observations today. It must also be neutral, otherwise star formation and evolution would have been disturbed in ways incompatible with observations [26], and the formation of "dark atoms" in the interstellar medium should have already been revealed in spectroscopy studies of heavenly objects [27]. However, micro-charges, about a million times smaller than the charge on the electron, can still be accommodated if only a few percent of the dark matter would have such charge [28]. Aside these generic constraints, there are still plenty of room for dark matter candidates with characteristics that avoid these limitations. And there are plenty of theoretical models that can accommodate them. Even though WIMPs are popular candidates because straightforward extensions of the Standard Model, like many flavours of super-symmetry, predict them [29], extremely light dark matter in the form of axion-like particles (ALPs) [30] or super-heavy dark matter [31,32] are also well justified. We will therefore not use the term WIMP in this review since many of the experimental results summarized later can be reinterpreted in terms of more general dark matter candidates. See [16,33-36] and references therein for recent reviews of candidates and production scenarios beyond the vanilla WIMP miracle.

Accumulations of dark matter are expected in halos around galaxies, including the Milky Way, and in galaxy clusters. In order to detect signatures from these regions with high density of dark matter, the dark matter particles need to be able to annihilate between themselves, or decay. It is the stable standard model particles that result from these processes that constitute the potential signature for indirect searches. These can be neutrinos, photons, electrons, protons or even light nuclei like He and $\mathrm{D}$ (and their antiparticles).

The distribution of dark matter in the halos of galaxies is an important ingredient both in calculating expected observables and eventually in interpreting a signal [37,38]. We know from N-body simulations of galaxy formation that galaxies are embedded in clumpy, rather complex dark matter halos that extend well beyond the visible galaxy [39]. There have been several proposals to describe the dark matter density distribution in galactic halos as a function of the distance from the centre of the galaxy [40-45]. The common feature of these profiles is a denser, typically spherically symmetric, region of dark matter in the centre of the galaxy, with decreasing density as the radial distance to the centre increases. Figure 2 shows a few examples of these profiles. Note that the slope of the density distribution varies with the distance, deviating from initial calculations in [40] that predicted a single power law distribution across the whole distance range. Where the different models diverge is in the predicted shape of the central region. Profiles obtained from N-body simulations of galaxy formation and evolution with a collisionless dark matter contribution tend to predict a steep power-law type behaviour of the dark matter component in the central region $[41,44]$, while profiles based on observational data (stellar velocity fields) tend to favour a more constant dark matter density near the core $[42,43,45,46]$. This discrepancy has been dubbed the core-cusp problem [47], and it is an unresolved issue. A recent fit to the motion of stars in the disk of the Milky Way [48] gives inconclusive evidence 
for a cored or cusped profile, reflecting on the observational difficulty in accurate mapping the dark matter distribution even of our galaxy. There are proposals within the dark matter paradigm that can ameliorate the problem, or even solve it, based on self-interacting dark matter models [49-53]. Self-interactions among dark matter particles while dark matter halos form will heat up the dark matter component, avoiding the otherwise strong collapse that forms cusped halos. The strength of the interactions (and therefore the mean free path of the dark matter particles between interactions) can be chosen to reproduce observations, including avoiding undesirable effects in early universe cosmology. Particle physics models describing the type of interactions that are allowed can be built under these simple assumptions. However, this solution still suffers from the fact that no dark matter candidates have been identified. A less exotic solution to the cusp problem could arise from the fact that one can actually describe the inner dark core from kinematic measurements of the visible inner region and global characteristics of the galaxy, like its viral mass [54]. This provides a measurement of the size of the dark matter core which is directly correlated to the luminous counterpart and that does not show any cuspy behaviour. A more radical claim is the recent study in [55] proposing that the cusp obtained in N-body simulations represents a numerical issue of the simulations themselves that do not reflect reality. Lacking further confirmation of the claims of the authors, their results can be taken to illustrate the difficulties and limitations involved in performing N-body simulations, even with today's powerful computers.

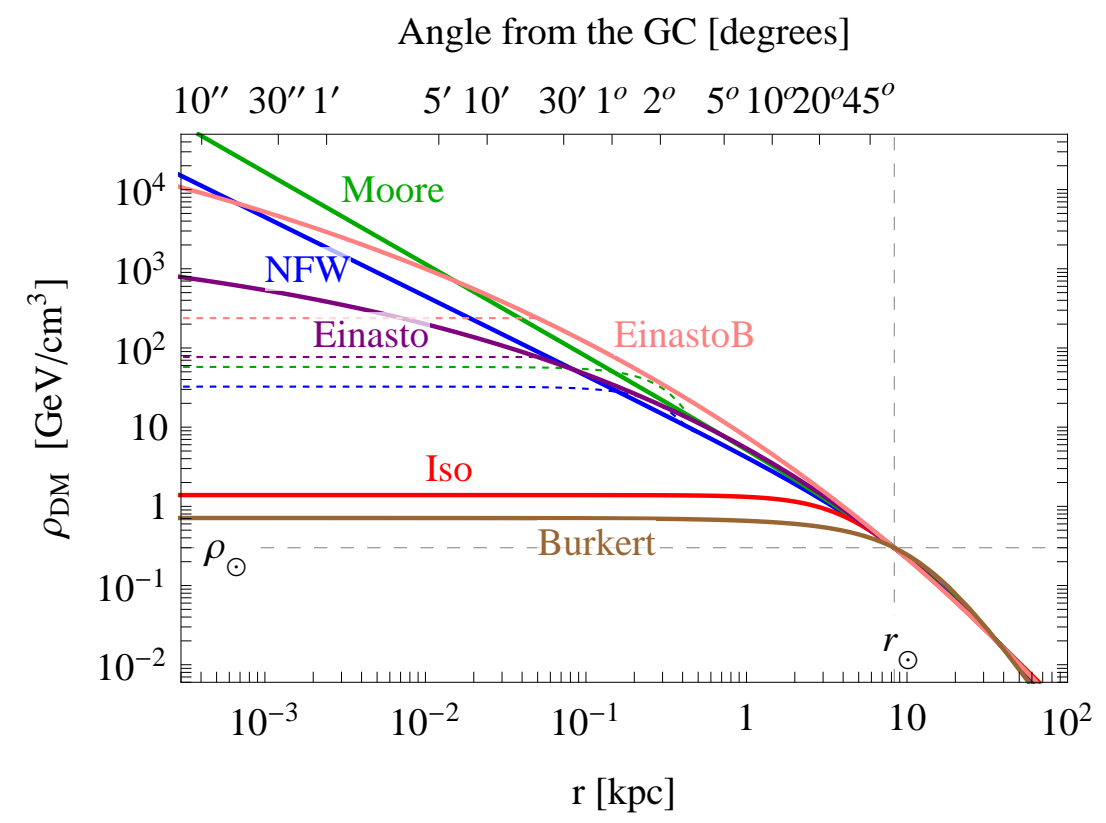

Figure 2. Commonly used Milky Way dark matter density profiles, including an isothermal model (Iso). See the reference below for details on the parameters used to obtain each curve. Here, suffice to show that the choice of one profile or another can have important consequences on the interpretation of experimental results. The distributions are normalized to a local density $\rho_{\odot}=0.3 \mathrm{GeV} / \mathrm{cm}^{3}$ in the solar neighborhood, at $r_{\odot}=8.3 \mathrm{kpc}$ from the Galactic Centre. Figure from [56], reproduced by permission of IOP Publishing. Copyright SISSA Medialab Srl. All rights reserved.

Following [57], a general parametrization of the dark halo in galaxies can written as (reference [57] has nothing to do with dark matter, but on the density distribution of visible mass in spherical galaxies. Equation (1) here is an adaptation of Equation (43) in that paper).

$$
\rho_{D M}(r)=\frac{\rho_{0}}{\left(\delta+\frac{r}{r_{s}}\right)^{\gamma} \cdot\left(1+\left(\frac{r}{r_{s}}\right)^{\alpha}\right)^{(\beta-\gamma) / \alpha}}
$$


where $r$ is the distance from the Galactic Centre, the parameters $\alpha, \beta$ and $\gamma$ determine the shape of the halo and $\rho_{0}$ is a normalization constant depending on the model, i.e., depending on the choice of $\alpha$, $\beta$ and $\gamma$. Different combinations of these parameters can recover the halo profiles mentioned above. These profiles describe the smooth distribution of dark matter around galaxies. Any substructure (clumpiness) must be added on top in order to describe the outcome of $\mathrm{N}$-body simulations $[58,59]$. The effect of clumpiness is usually parametrized as a multiplicative boost factor to the expected signal from annihilations without substructure $[60,61]$ and it is, by necessity, an average of the enhancement given by simulations of a population of halos.

Figure 2 shows that the different halo models proposed show agreement on the dark mater distribution at the location of the Solar System (about $8 \mathrm{kpc}$ from the Galactic Centre) and beyond, but the predicted dark matter density distribution diverges considerably at distances near the centre of the galaxy [62]. This is of course also true when considering other galaxies and assuming the same type of profiles apply universally at any redshift. However, universality might not be completely justified, and the shape of a dark halo can depend on local characteristics of any given galaxy. There are indications that halo profiles might depend both on the size of the galaxy and the structure of its surroundings, since the original mass function of nearby satellites will affect the accretion of additional dark matter to the halo, causing the inner slope of the dark matter profile to deviate form a universal function like the ones in Equation (1) [63]. Indeed, recent results from the Gaia mission point to a more complex dark matter halo in the Milky Way than just a spherical distribution. Gaia will map the position and radial velocity of about one billion stars, providing and unprecedented 3-dimensional picture of our galaxy and invaluable data to precisely map the dark matter halo for the first time. Gaia's data release 2, along with data from the Sloan Digital Sky Survey (SDSS), have already provided indications that our dark matter halo has a non-trivial structure, with localized substructures due to the recent history of our Galaxy that might not yet be in equilibrium. Actually, the Gaia results seem to be in tension with the characteristics of the dark halo derived from previous stellar data [64-68]. The first implication of these results is that the Milky Way halo is more complex than initially thought, with local substructure that now can be mapped. This can have implications for direct dark matter searches whose expected signal depends on the assumed dark matter density in the solar neighborhood.

However, in the bigger picture, the Gaia results point to a dark matter halo which is strongly dependent on the specific history of the galaxy, determined by past accretion events from nearby satellite galaxies. If dark matter halos strongly depend on the individual history of each galaxy, the assumption of universality of the dark matter profiles breaks down, with consequences for the interpretation of indirect dark matter searches as well. In particular there are two related aspects to take into account: The integrated rate of supernova explosions through the evolutionary history of a galaxy and the role of baryon feedback in the star formation cycle of the galaxy [37,69-72]. The ejecta from supernova explosions can heat the surrounding interstellar gas, injecting energy into it to a point that further star formation due to gravitational collapse significantly slows down, or even stops. As the affected gas expands and cools down, gravity draws it back towards the center of the galaxy, reinitiating the star formation process and eventually leading to more supernovae explosions. For large galaxies, with a large amount of gas, the cycle from star formation to explosion to re-ignition of star formation can be quite fast. During these processes the baryonic mass distribution of the galaxy fluctuates, inducing a change in the gravitational potential of the gas which can have an effect on the dark matter distribution. In particular, this process could solve the core-cusp problem, since the net effect on the dark matter component is to redistribute the dark matter away from the galactic center, converting an initially cusped profile in a cored profile. The rate of supernova ejecta and the strength of baryon feedback, and their effect on the dark matter distribution of a galaxy, depend on the size, age, evolution and surroundings of the galaxy, which support the idea that universal halo functions are just a first approximation to the structure of a galaxy, but that individual characteristics need to be taken into account. This can affect the calculations of $J$-factors discussed below. 
Barring the caveats just mentioned, given a specific dark matter halo, the flux of cosmic-rays, gamma-rays or neutrinos arising from dark matter annihilations in a given object can be expressed as

$$
\frac{d N_{i}}{d A d t d E_{i}}=\frac{d \Phi_{i}}{d E_{i}}=\frac{1}{4 \pi} \frac{\left\langle\sigma_{\mathrm{A}} v\right\rangle}{2 m_{\mathrm{DM}}^{2}} \frac{d N_{i}}{d E_{i}} \times \int_{\Omega} \int_{\text {l.o.s. }} \rho_{D M}^{2}(r) d r d \Omega
$$

where $i$ stands for the type of particle, $\left\langle\sigma_{\mathrm{A}} v\right\rangle$, is the thermally averaged product of the dark matter self-annihilation cross-section times the dark matter velocity, $d N_{i} / d E_{i}$ is the spectrum of species $i$ produced by the annihilations, $\rho_{D M}$ is the dark matter density of the source, given by Equation (1) and $m_{\mathrm{DM}}$ is the dark matter mass. The integral of the squared density along the line of sight (l.o.s.) is the so-called J-factor. As argued above, the J-factor is source-specific, and encompasses our knowledge of the structure of the dark halo of the source in consideration. The J-factor depends on the halo profile chosen as well as on the distance to the source, and results from indirect dark matter searches must be given under the assumption of a specific halo model. Clearly, distances need to be estimated independently, which for far away objects can add to the final uncertainty of the result. J-factors are measured in $\mathrm{GeV}^{2} \mathrm{~cm}^{-5} \mathrm{sr}$. As Figure 2 illustrates, using one parametrization of $\rho_{D M}$ or another can give results that differ by as much as orders of magnitude.

Assuming a particle physics model which gives the expected particle spectrum, $d N_{i} / d E_{i}$, and a halo model, then an experimental measurement of $d \Phi_{i} / d E_{i}$ can be used to probe the two independent terms in Equation (2): $\left\langle\sigma_{\mathrm{A}} \mathrm{v}\right\rangle$ versus the dark matter mass, $m_{\mathrm{DM}}$. Experimental efforts are usually kept as model-independent as possible, looking for signatures from generic candidates over a few benchmark masses that cover a wide mass range and, since the exact branching ratios of dark matter annihilations into different channels are unknown, analyses are typically optimized assuming $100 \%$ annihilation to a few characteristic channels. This brackets the expected spectrum from any realistic model which would produce an spectrum that is a weighted mixture of all the possible annihilation channels.

If signals from dark matter decay, instead of annihilation, are to be probed, then Equation (2) needs to be slightly modified: The l.o.s. integral is over $\rho$ and not $\rho^{2}$ (only one particle participates in a decay, while two are needed in an annihilation), only one $m_{\mathrm{DM}}$ factor is needed in the denominator (for the same reason), and what is probed is $1 / \tau$ instead of $\left\langle\sigma_{A} v\right\rangle$, where $\tau$ is the candidate lifetime. In this case the units of the $J$-factor are $\mathrm{GeV} \mathrm{cm}^{-2} \mathrm{sr}$.

It is worth pointing out that the term $\left\langle\sigma_{\mathrm{A}} v\right\rangle$ can provide more information than it might seem at first sight. It is not only sensitive to a given particle model, through what the model predicts for $\sigma_{\mathrm{A}}$, but it is also sensitive to the structure of the host galaxy (or the medium where the annihilations take place), through the velocity distribution from which the annihilating particles are drawn. For $2 \rightarrow 2$ annihilations in the non-relativistic case, the term $\left(\sigma_{\mathrm{A}} v\right)$ can be expanded in powers of angular momentum in the square of the relative velocity of the particles as $\sigma_{\mathrm{A}} v=\sigma_{s}+\sigma_{p} v^{2}+\ldots$, where $\sigma_{s}$ and $\sigma_{p}$ are constants (see [73] for the corresponding relativistic expression and a thorough discussion on the applicability regimes of $\left.\left\langle\sigma_{\mathrm{A}} v\right\rangle\right)$. The previous expression explicitly shows the constant behaviour of s-wave $(l=0)$ scattering and the quadratic dependence on the relative velocity of p-wave $(l=1)$ scattering. Given the dark matter velocity in the halos of galaxies, $\beta \sim 10^{-6}$, the relative velocity of two annihilating particles is typically low enough to make the p-wave annihilation rate subdominant to the s-wave term. However, s-wave annihilations to some channels (fermions for example) can be chirality suppressed by a factor $m_{f} / m_{\mathrm{DM}}$, where $m_{f}$ is the fermion mass, so the prospects of indirect detection of certain dark matter candidates can become slim. There are, however, natural ways out of this problem that bring back the hope for some of the indirect detection channels. One is simply to search for dark matter annihilations in objects which are violent enough for dark matter to orbit with high velocities, where p-wave annihilations naturally dominate [74]. Further, if one considers annihilations with three-body final states, $2 \rightarrow 3$, where the third particle arises as a $\gamma, \mathrm{Z}$ or $\mathrm{W}$ bremsstrahlung in the final state, the helicity suppression is highly ameliorated. Besides, these type of annihilations can simultaneously produce photons and electrons, or photons and neutrinos, providing a multi-messenger signal from the same process. An interesting study case for experimentalists. 
Another way to avoid the suppression of s-wave annihilations is through the Sommerfeld enhancement in models with self-interactions in the dark matter sector [75-80]. In such case the new potential enhances the s-wave cross section through the well known Sommerfeld effect [81] by factors as high as three orders of magnitude, making such models testable with the messengers and sources mentioned in the sections below.

There is a further point to consider concerning the use of Equation (2). As it has been presented it includes only the spectrum from the source under consideration but it ignores the diffuse cosmological contribution from all the sources along the line of sight, at different redshifts. This contribution can be sometimes safely neglected, for example in searches from nearby objects like the center of the Milky Way where the additional signal from the far cosmos is subdominant. However, it can be of relevance when pointing at extended sources or in analyses with poor angular resolution. In case this effect needs to be included, a cosmological model needs to be assumed and energies detected at the Earth need to be properly redshifted, and an integral over redshift performed. These effects have been clearly described, for example for gamma-rays and neutrinos, in [82,83]. Diffuse cosmological contributions can also be important when considering halos with substructure. Since dark matter annihilations scale as the dark matter density squared, high-redshift but clumpy halos could contribute a non-negligible signal at Earth.

\section{Dark Matter Signatures from the Cosmos}

Since the expected signal from dark matter annihilation (decay) is proportional to $\rho_{D M}^{2}\left(\rho_{D M}\right)$, natural sources to consider in indirect searches are regions in the universe with an increased density of dark matter. Typically, our own Milky Way, close-by dwarf spheroid galaxies and galaxy clusters are considered in indirect searches. Dwarf spheroid galaxies are good candidates because they present high mass-to-light ratios, with little interstellar gas, and they seem therefore to be dominated by dark-matter. Additionally, there is no evidence of non-thermal emission from any of the 20+ known dwarf spheroids that could produce a background of high energy neutrinos, $\gamma$-rays or cosmic-rays so they are a clean target from the observational point of view. Even if they are small systems, with J-factors much smaller than our Galactic Centre, observations of several dwarves can be stacked in order to increase the sensitivity to a potential dark matter signal.

Indirect searches for dark matter have indeed been performed by gamma-ray telescopes (MAGIC, e.g., [84-86], H.E.S.S., e.g., [87-90], VERITAS [91,92]), X-ray telescopes (XMM-Newton, e.g., [93-96], NuSTAR, e.g., [97-99], Suzaku, e.g., [100-103]), cosmic-ray detectors (HAWC [104-106]), detectors in space (Fermi-LAT, e.g., [107-109], DAMPE, e.g., [110], CALET [111], AMS [112]) and neutrino telescopes (IceCube, e.g., [113-115], ANTARES, e.g., [116-118], Baikal, e.g., [119-121], Baksan [122] or Super-Kamiokande, e.g., [123-125]). Many of these detectors have upgrade programs underway, in different stages of R\&D or implementation (CTA [126], IceCube-Gen2 [127], KM3NET [128], Baikal-GVD [129], Hyper-Kamiokande [130]), which will bring indirect dark matter searches to the next level in mass coverage and sensitivity.

Each messenger has its quirks and features, though, both from the point of view of propagation and/or detection. In order to increase the sensitivity to specific sources that can be observed with different instruments, several collaborations have joined forces and obtained limits from their combined observations. Combining results from different experiments requires a consensus on the use of common $J$-factors for each target, statistical techniques and the treatment of the systematic uncertainties of the different instruments. The result of these efforts are usually stronger limits than those obtained by the individual collaborations. They also help unify analysis methods and the use of common external inputs (astrophysical quantities, cross sections, dark matter models...) across the community, which in the long run helps obtaining a coherent view of the field and makes it easier to compare, and even to combine, results from different messengers. One can really talk of a multi-messenger era in dark matter searches. 


\subsection{Gamma- and X-Rays}

Gamma-rays propagate without deflection, so they point to their source, and are relatively easy to detect, the technology being available since long. Gamma-ray telescopes and gamma-ray detectors on space are able to point with great accuracy (about 0.1 degree for single photons above $10 \mathrm{GeV}$ in Fermi-LAT) and the same for Cherenkov telescopes like MAGIC or H.E.S.S. for example (although at higher energies, above about $100 \mathrm{GeV}$ ) and they cover a wide range of photon energies, from $20 \mathrm{GeV}$ in Fermi-LAT to few hundred TeV in Cherenkov telescopes. However, absorption and backgrounds are always a concern when searching for new physics with photons from space. The Milky Way centre is a quite crowded region with $\gamma$-emitting objects and a diffuse component from unresolved sources and star formation regions. In this respect, the clean structure of dwarf spheroid galaxies is expected to provide a favourable environment for dark matter searches with gammas, and indeed strong limits on the dark matter annihilation cross section have been obtained from these objects. An illustration of the results that can be obtained with this kind of searches is shown in the left plot of Figure 3. The figure shows $95 \%$ confidence level upper the limits on the velocity-averaged dark matter annihilation cross section as a function of dark matter mass obtained from observations of $45 \mathrm{dwarf}$ galaxies with the Fermi-LAT telescope [109]. Refined limits can be obtained exploiting the different energy reach (and therefore dark matter mass sensitivity) of different instruments, as it is shown in the right plot of Figure 3. The plot shows a combined limit on $\left\langle\sigma_{A} v\right\rangle$ using results from Fermi-LAT, HAWC, H.E.S.S., MAGIC and VERITAS, and reaches an improvement of up to one order of magnitude for masses above $10 \mathrm{TeV}$ due to the contribution of the Cherenkov telescopes [131].
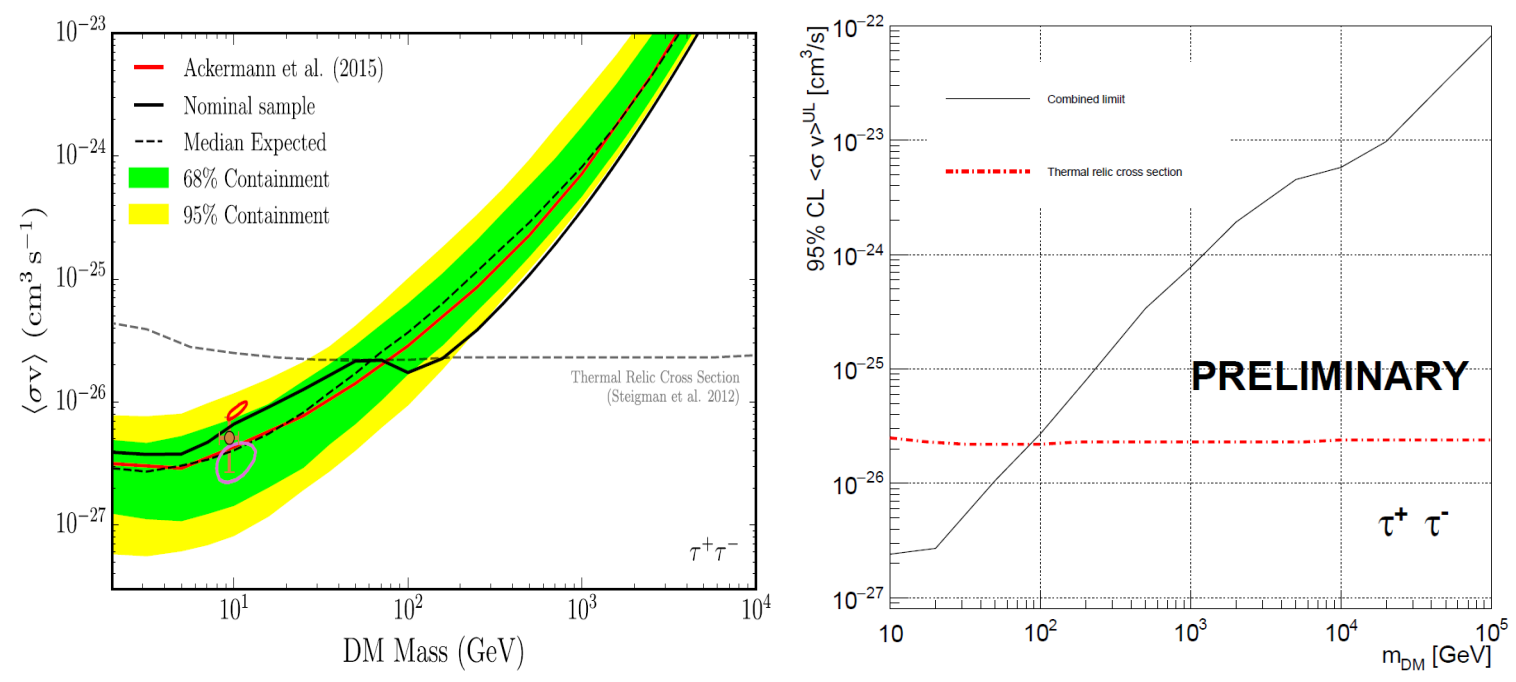

Figure 3. (Left) The 95\% confidence level upper limits on the velocity-averaged dark matter annihilation cross section obtained from 45 dwarf galaxies with the Fermi-LAT instrument, assuming 100\% annihilation to $\tau^{+} \tau^{-}$. The yellow and green lines represent the $95 \%$ and $68 \%$ quantiles respectively. The solid red line shows the limit from a previous analysis using 15 sources. The closed contours and marker show the best-fit regions (at $2 \sigma$ confidence) of several dark matter interpretations of the Galactic Centre excess. Figure reprinted with permission from [109]. Copyright 2020 AAS. (Right) The 95\% confidence level upper limits on the dark matter annihilation cross from the combination of data from observations of 20 dwarf galaxies by Fermi-LAT, HAWC, H.E.S.S., MAGIC and VERITAS, assuming $100 \%$ annihilation to $\tau^{+} \tau^{-}$. Figure from [131].

The uncontroversial negative results from dwarf galaxies can be confronted with the status of dark matter searches with gammas from the Galactic Centre. An intriguing excess of gamma-rays in the few $\mathrm{GeV}$ region over the background expected from conventional sources has been claimed by several authors, both from EGRET data [132] and more recently from Fermi-LAT data [133]. However, an excess is of course defined with respect to a background, and here is where difficulties arise. The region around 
the Galactic Centre is one of the more complex in the sky, not least in gamma-rays. It is a star-forming region, surrounded with interstellar gas and with plenty of individual sources, like short-period pulsars and supernova remnants, as well as allegedly unresolved sources. Gamma-rays can be produced in point sources but also as a diffuse glow by electron bremsstrahlung, by inverse Compton scattering of electrons and positrons on the intense ambient radiation field in the region, or as products of the interactions of cosmic-rays with the interstellar gas. The calculation of the expected gamma-ray flux from all these processes, along with the galactic model used to track the diffusion of cosmic-rays in their way through the galaxy, is plagued with uncertainties which reflect in the predicted gamma ray emission from the Galactic Centre (which is the background when searching for dark matter) [134-137]. Indeed the EGRET excess was promptly explained by detailed calculations of the expected cosmic-ray flux without the need of new physics [138]. An additional important uncertainty in modeling the inner region of the Galaxy arises from the "Fermi bubbles" [139]. Their contribution to the gamma ray glow is difficult to estimate since information is lacking about their structure and emitted spectrum close to the Galactic Centre. Masking known point sources and subtracting the diffuse emission from the Galactic Centre in order to characterize the excess is therefore a really complicated task, plagued with unknowns. However, a new method of analyzing photon sky maps, non-Poisson template fitting, has been proposed in [140] and applied to the inner galactic region. The method is more sensitive to unresolved point sources than traditional Poisson-count based analyses, and shows that the Fermi-LAT gamma-ray excess can be explained by the background emission produced by a population of sub-threshold objects, without the need of dark matter.

However, the location of the excess, within $1.5^{\circ}$ of the Galactic Centre, is indeed a place where an increased density of dark matter is expected. Given the unknowns in any dark matter model, the excess can of course be also fitted as originating from dark matter annihilations, as indeed it has (see, e.g., [141-145] although the literature is vast on this topic). The main "result" of these claims is that a dark matter particle in the mass range of a few tens of $\mathrm{GeV}$ to a few hundred $\mathrm{GeV}$, annihilating to soft (typically $b \bar{b}$ or $\tau^{+} \tau^{-}$) or hard (typically $W^{+} W^{-}$or $t \bar{t}$ ), channels can fit the data. The wide range of masses and annihilation channels proposed simply reflects the underlying uncertainties in estimating the background, as well as the rather unconstrained options that the dark matter solution provides. The allowed parameter space in dark matter models is ample enough to be able to fit the Fermi-LAT gamma ray excess without problem, even with the oversimplifying assumption of a single one-particle species annihilating through just one channel and taking into account limits from new particle searches in accelerators.

The authors in [135] have performed an extensive study of the Galactic Centre and used several models of the galaxy to estimate the expected gamma ray glow, finding that an excess over the expected gamma ray flux from the Galactic Centre can be obtained in all the models considered. However, they also point out that the excess is still compatible, given the huge uncertainties, with the emission from standard astrophysical sources when considering contributions from cosmic-ray interactions in the interstellar medium or unresolved populations of pulsars. It is, of course, also compatible with ad-hoc dark matter models but, again, Occam's razor renders at this point the dark matter interpretation of the gamma ray excess from the Galactic Centre as, at most, a mere viable solution, but not the only one. A summary of this study, in comparison with the original claims of the excess, is shown in Figure 4. The figure shows the expected emission from the Galactic Centre obtained by the Fermi-LAT collaboration with a detailed modeling of the Galaxy (points tagged as "Sample") compared with the excess claimed by other authors, using different assumptions on the morphology of the Galaxy and background templates (so, in a strict sense, the curves are not really directly comparable). The shaded area represents the systematic uncertainty from the Fermi-LAT collaboration analysis, and it is obtained as the envelope of the predictions of the gamma-ray flux under different assumptions on diffuse gamma-ray emission, galaxy morphology, gas distributions in the galaxy or cosmic-ray distribution and propagation, but, in any case, without any new physics. A similar conclusion is reached in [107], where diffuse galactic emission, inverse-Compton emission and a central source of 
electrons can explain the Fermi-LAT data without a dark matter component, practically ruling out thermal dark matter as the explanation of the galactic centre gamma-ray excess.

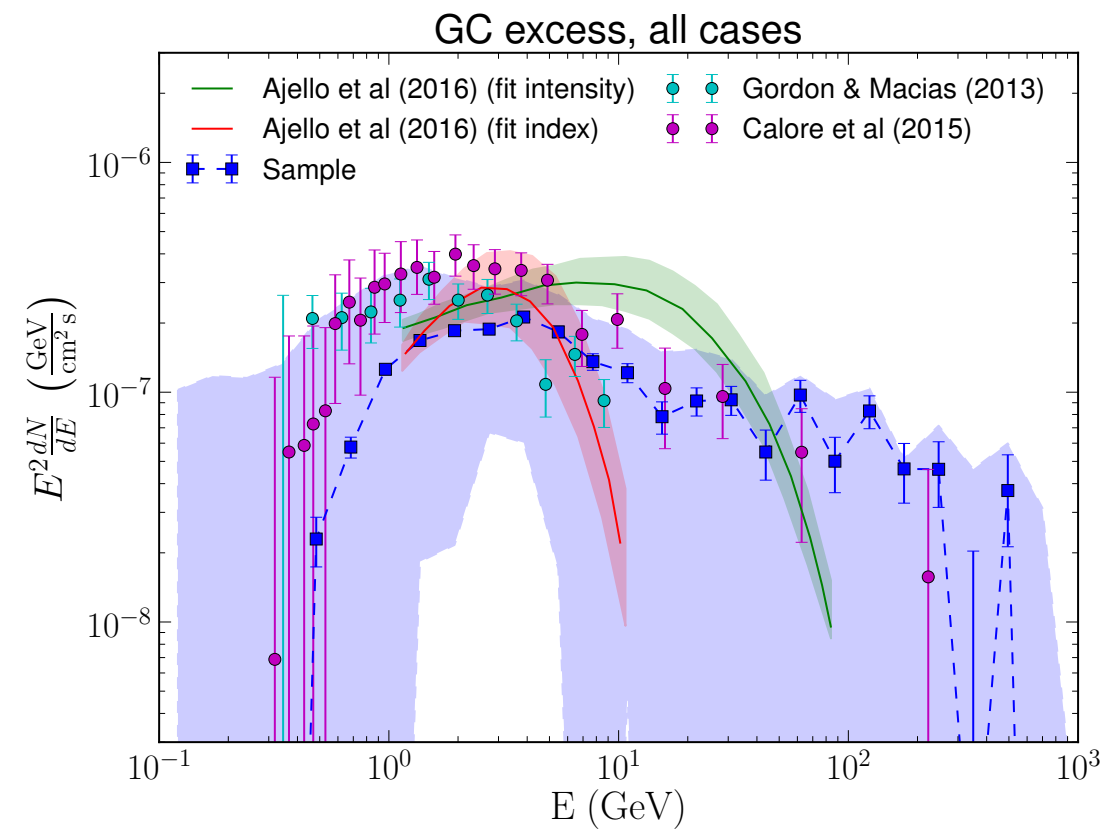

Figure 4. Spectrum of the Galactic Centre excess. The systematic uncertainty band shows the envelope of different Galactic Centre excess fluxes obtained using different models of diffuse gamma-ray emission and source masking. Figure reprinted with permission from [135]. Copyright 2020 AAS. See reference for details of the analyses mentioned in the inset caption.

While the rather energetic gamma-rays can probe the $\left(\left\langle\sigma_{\mathrm{A}} v\right\rangle, m_{\mathrm{DM}}\right)$ parameter space for dark matter masses of $O(\mathrm{few} \mathrm{GeV})$ and above, the softer diffuse X-ray sky can be used to probe dark matter masses down to the $\mathrm{keV}$ region $[146,147]$. X-rays can be produced directly from low mass dark matter annihilations or decays, but they can also be produced by inverse Compton scattering of $e^{+} e^{-}$from the annihilations or decays of dark matter of any mass on background photons. Inverse Compton scattering of charged annihilation or decay products on $\mathrm{CMB}$ photons, dust or starlight will produce a diffuse photon flux with typical photon energies of the order of $1-100\left(m_{\mathrm{DM}} / 10 \mathrm{GeV}\right)^{2} \mathrm{keV}$ [147], where $m_{\mathrm{DM}}$ is the dark matter mass, and the energy range covers therefore the $\mathrm{X}$-ray region. $\mathrm{X}$-ray telescopes provide, therefore, a complementary way to search for dark matter from galaxy clusters and galaxies, including the Milky Way. One of the best theoretically justified light dark matter candidate in the keV mass region is a right-handed (i.e., sterile) neutrino, which can be accommodated in extensions of the standard model without many additions to the particle spectrum of the theory [148-151]. Sterile neutrinos can be produced in the early universe through mixing with active neutrinos, so the mass $M_{S}$ of the sterile neutrino and the mixing angle with active neutrinos are two fundamental parameters of any model. Probing the $\left(M_{s}, \sin ^{2}(2 \theta)\right)$ parameter space amounts to probing the feasibility of sterile neutrinos to be dark matter. Sterile neutrinos have a radiative decay channel into an active neutrino and a photon $\left(v_{s} \rightarrow v \gamma\right)$. The integrated effect of decays at different redshifts during the evolution of the universe would result in a broad X-ray band today on top of the the diffuse X-ray astrophysical background. The non-detection of such feature has already allowed to constrain the $\left(M_{s}, \sin ^{2}(2 \theta)\right)$ parameter space of sterile neutrino dark matter with X-ray telescopes [93-95,99-102,152-154]. The left plot of Figure 5 shows that X-ray telescopes alone have a strong constraining power on the $\left(M_{s}, \sin ^{2}(2 \theta)\right)$ plane, in this case illustrated on the $v$ MSSM model, a minimal extension of the standard model that includes neutrino masses and which incorporates a dark matter candidate in the form of a right-handed neutrino of a few keV [155]. Additionally, the same type of analyses can be used to constrain the annihilation cross section of a generic low-mass dark matter candidate. The right plot of Figure 5 shows the limit 
on the velocity averaged annihilation cross section of a generic light weakly interacting dark matter candidate assuming annihilation to two photons obtained with the same X-ray observations as in the left plot. While this result has been obtained for a quite favourable situation for X-ray detectors, direct annihilation into photons, the limit on $\left\langle\sigma_{\mathrm{A}} v\right\rangle$ is quite strong if compared with the results from other messengers.
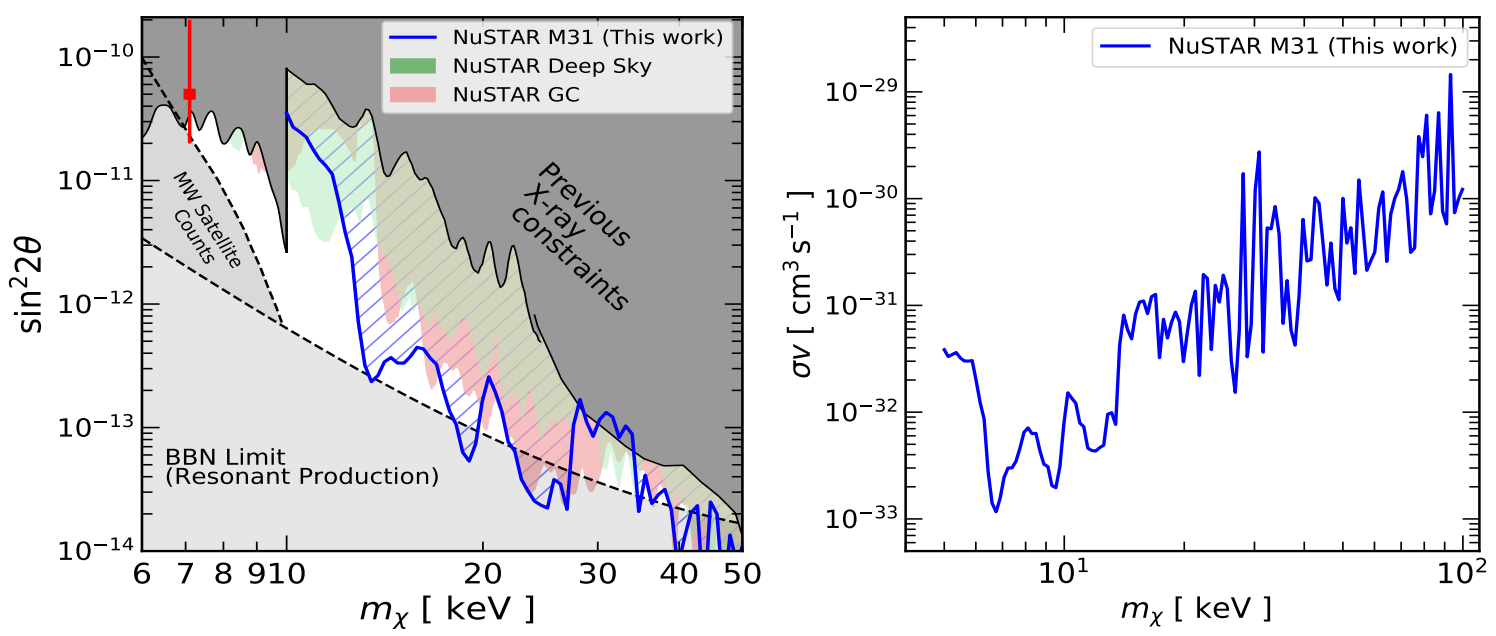

Figure 5. (Left) Excluded areas of the sterile neutrino mass and mixing angle parameter space from NuSTAR observations of M31, the Galactic Center and the deep X-ray sky. See reference below for details. (Right) Upper limit on weakly interacting dark matter annihilation cross section from NuSTAR observations of M31, assuming annihilation to two monoenergetic photons. Figures reprinted with permission from [153]. Copyright 2020 by the American Physical Society.

\subsection{Neutrinos}

Neutrinos also propagate undeflected and therefore point to their source, but they present additional challenges compared with photons. First, their extremely low cross section with matter means that large detectors and exposure times are needed to collect just an event from a given source, not to mention a handful of events that would be statistically significant by themselves. In this sense, the best bet of neutrino telescopes to function as dark matter detectors is to correlate their findings with other messenger or, ideally, more than one. This would, in turn, help interpreting any signal from gamma-rays or cosmic-rays, since an excess of events from a single messenger might be subject to interpretation as background from other processes rather than from dark matter, or it would need quite ad-hoc cooked theoretical models to explain why the dark sector annihilates or decays to some standard model particles but not others. Besides, neutrino detectors have a limited angular resolution, of the order of $1^{\circ}$ at $O(100 \mathrm{GeV})$ neutrino energy, only reaching below one degree at TeV energies, and therefore pointing becomes challenging in searches for relatively low-mass dark matter candidates. Even in a perfect detector the angular resolution would be ultimately limited by the physics of the neutrino-nucleon cross section. Limited pointing resolution impacts the degree of background rejection, mainly atmospheric neutrinos, when pointing towards a source. On the other hand, neutrinos have the advantage of not loosing energy or being absorbed through their propagation over cosmic distances. Neither there is a significant background or foreground from astrophysical objects, so they remain an attractive signature. They are indeed the only possible messengers to use when looking for dark matter accumulated in dense objects like the Sun or the Earth. More on this below.

The total number of signal events, $\mu_{\mathrm{s}}$, expected at a neutrino telescope is given by,

$$
\mu_{\mathrm{s}}=T_{\text {live }} \sum_{\alpha} \int \mathrm{d} \Omega \int \mathrm{d} E_{v} A_{v_{\alpha}}^{\text {eff }}\left(\Omega, E_{v}\right) \Phi_{v_{\alpha}}\left(E_{v}\right),
$$


where $T_{\text {live }}$ is the exposure time, $A_{v_{\alpha}}^{\text {eff }}\left(\Omega, E_{v}\right)$ the detector effective area for neutrino flavour $\alpha$, that depends on the detector response with respect to the observation angle and neutrino energy and $\Phi_{v_{\alpha}}\left(E_{v}\right)$ is the neutrino flux for flavour $\alpha$ arising the annihilation processes. Note that the effective area of neutrino telescopes is not a fixed quantity, but it depends not only on neutrino energy and arrival direction, but also on the specific event selection performed. In the absence of a signal, the $90 \%$ confidence level limit on the number of signal events, $\mu_{\mathrm{s}}^{90}$, can be directly translated into a limit on the neutrino flux and, in turn, into a limit on either the velocity-averaged annihilation cross section $\left\langle\sigma_{\mathrm{A}} v\right\rangle$ or the dark matter lifetime as a function of dark matter mass, through Equation (2). As in the case with gamma-rays, sources with similar characteristics, where the signal can be expected to be also similar, can be stacked to increase the signal-to-noise ratio. This is typically done when considering nearby dwarf galaxies.

The current status of searches for dark matter with neutrinos from galaxies span a large chunk of dark matter masses as illustrated in Figure 6. The figure shows the current landscape of limits on the dark matter self-annihilation cross section as a function of dark matter mass obtained by the main players in the neutrino dark matter search industry, including many low-energy experiments which are not the focus of this review [156]. Next-generation experiments are shown as dashed-lines, while the shaded areas show current limits, some of them obtained independently from the collaborations by the authors (marked with a heart). Results cover an impressive eleven orders of magnitude in dark matter mass, extending beyond the theoretical limit for a thermal relic, where results should be interpreted under different class of models than the vanilla WIMP scenario (see e.g., [33] and references therein).

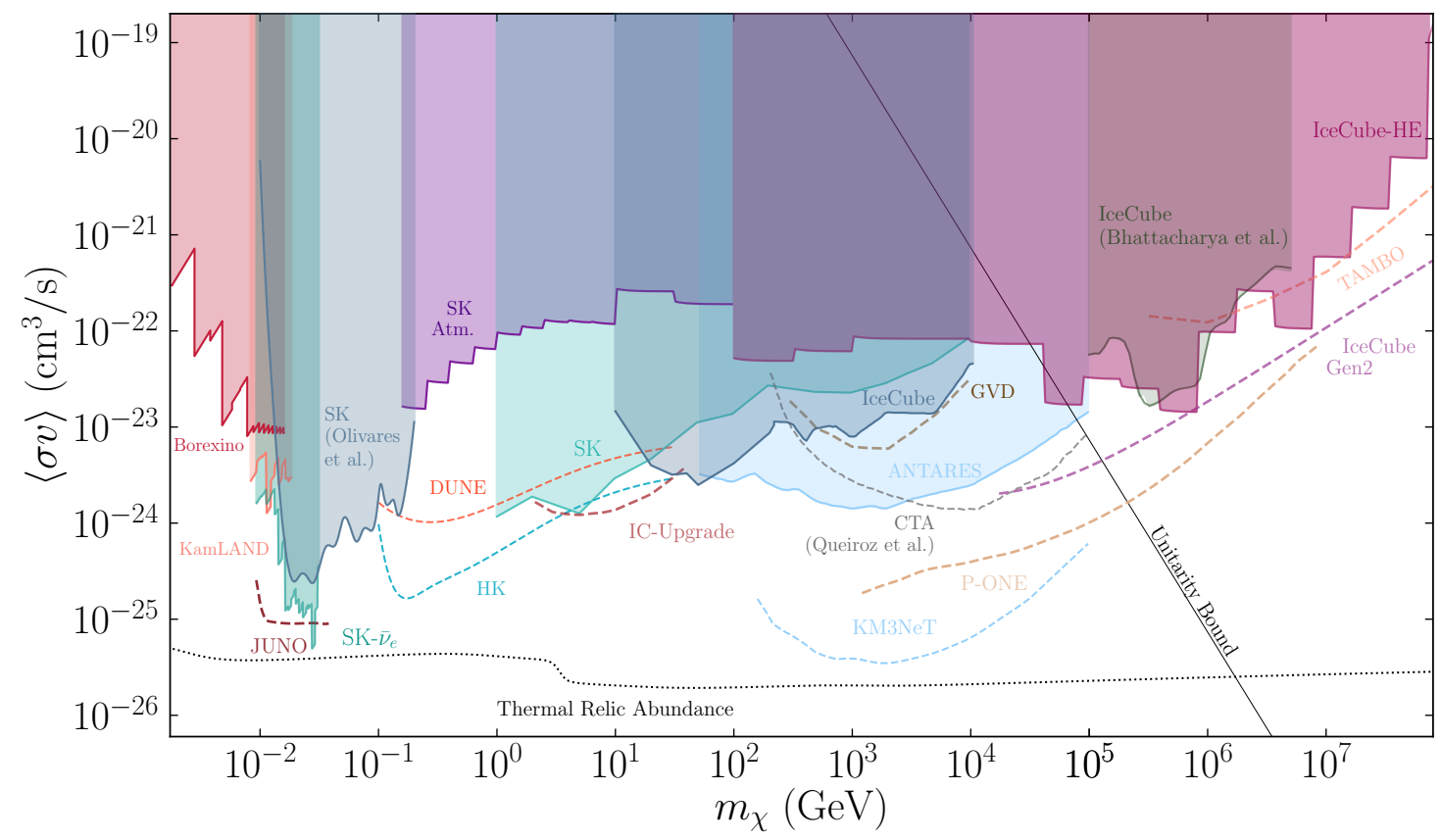

Figure 6. Summary of results on the velocity weighted dark matter annihilation cross section from different experiments. Solid lines show limits, dashed lines sensitivities of future facilities assuming five years data taking ( $100 \mathrm{~h}$ of observation for the CTA sensitivity). The heart symbols represent analyses performed by the authors of [156] with public data, and not by the collaborations. Figure from [156].

In addition to our Galaxy and other objects at cosmological distances, the Sun and the centre of the Earth are also two possible sources of dark matter annihilations that could be detectable due to their proximity. The effect of the Sun gravitational attraction over the lifetime of the Solar System can result in the capture dark matter from the local halo through repeated scatterings of dark matter particles in orbits crossing the star [157-161]. The same applies for the Earth [162,163]. The only caveat is that only neutrinos from dark matter annihilations in the centre of these objects can escape their 
dense interior. So it is only neutrino telescopes that can pursue dark matter searches from the centre of the Sun and Earth (with some exceptions mentioned below).

The expected neutrino flux from dark matter annihilations inside the Sun depends, among other factors, on the capture rate of dark matter (which is proportional to the dark matter-nucleon cross section), and the annihilation rate (which is proportional to the velocity-averaged dark matter annihilation cross section). The evolution of the number density of dark matter particles, $N_{\mathrm{DM}}$, accumulated in the Sun interior follows the equation

$$
\frac{d N_{\mathrm{DM}}}{d t}=\Gamma_{\mathrm{C}}-2\left\langle\sigma_{\mathrm{A}} v\right\rangle\left(N_{\mathrm{DM}}^{2} / 2\right)
$$

where $\Gamma_{C}$ is the capture rate per unit volume. The numerical factors take into account that annihilations consume two particles per interaction but there are only $1 / 2$ of the particles available to form pairs. We have neglected a possible "evaporation" term, proportional to $N_{\mathrm{DM}}$, which is only relevant for very small dark matter masses, $\lesssim 4 \mathrm{GeV}[160,164,165]$, below the range currently probed by neutrino telescopes due to their relatively high energy threshold (precisely a few $\mathrm{GeV}$ ), but that can be an issue to take into account in future low-energy extensions of current projects. Equation (4) has an equilibrium solution given by

$$
N_{\mathrm{DM}, \mathrm{eq}}=\sqrt{\frac{\Gamma_{\mathrm{C}}}{\left\langle\sigma_{\mathrm{A}} v\right\rangle}}
$$

which represents the steady amount of dark matter accumulated in the Sun if capture and annihilation have reached equilibrium. Since the Sun is around $4.6 \mathrm{Gyr}$ old, it is usually assumed that equilibrium has indeed been achieved. In this case, the neutrino emission only depends on the capture rate, which is directly related to the dark matter-nucleon scattering cross section, $\sigma_{\mathrm{DM}-\mathrm{N}}$, and this allows to set limits on $\sigma_{\mathrm{DM}-\mathrm{N}}$ from the measured (or rather, lack of) anomalous high-energy neutrino flux from the Sun. In models where equilibrium might not have been achieved, then an assumption on the dark matter self annihilation cross section is necessary to derive predictions on the neutrino signal from dark matter annihilations.

The dark matter-nucleon cross section can be expressed in terms of a spin-dependent, $\sigma_{\mathrm{SD}}$, and spin-independent, $\sigma_{\mathrm{SI}}$, contributions [166] and, since the Sun is mainly composed of Hydrogen (75\% of $\mathrm{H}$ and $24 \%$ of $\mathrm{He}$ in mass) [167], the capture of dark matter from the halo by the solar gravitational well occurs mainly through the spin-dependent scattering. Heavier elements constitute less than $2 \%$ of the mass of the Sun, but can still play a role when considering dark matter capture, since the spin-independent cross section is proportional to the square of the atomic mass number. These heavy elements can also take part in the spin-dependent capture process if dark matter presents momentum-dependent interactions with normal matter, giving rise to an increased capture rate in comparison to previous calculations, that can have a bearing in the interpretation of experimental results [168]. Even if searches for dark matter with neutrinos from the Sun are performed in the most model-independent way, there is of course the possibility to probe specific models, and both the main collaborations or external authors using public data have done so. These include limits on Kaluza-Klein dark matter arising in models of universal extra dimensions [169], inelastic dark matter [170], strongly interacting dark matter [171], or specific extensions of the MSSM [172-174], among others.

There are some theoretical scenarios that predict a flux of gamma-rays or cosmic-rays due to dark matter annihilations in the Sun. If the dark matter "world" is coupled to our usual world through long lived mediators that can escape the Sun interior and decay into Standard Model particles in-flight to the Earth, then other techniques can also search for dark matter accumulated in the Sun. In such models of secluded dark matter [175], an excess of gamma-rays or cosmic-rays from the direction of the Sun could also be interpreted as a signature of dark matter annihilations inside it [176-179]. Secluded dark matter models also present advantages for neutrino searches. Neutrinos loose energy on their way out of the dense solar interior, so the flux that would reach the Earth from annihilations in the solar core would be mainly low-energy (up to a few $100 \mathrm{GeV}$ ) independent of the mass of the dark matter annihilating 
inside the Sun. The correlation between dark matter mass and highest possible neutrino energy is therefore lost. However, since the decay of the mediator in secluded models can occur outside the Sun, the neutrinos will reach the Earth without loosing energy. This is advantageous for neutrino telescopes, which usually have better sensitivities and pointing for higher neutrino energies, $O(100 \mathrm{GeV})$ and above. These models add, of course, additional free parameters: The mediator mass, lifetime and decay branching ratios to different final states. Experimental results are therefore usually provided under different assumptions for the mediator mass and lifetime, for a given annihilation channel $[180,181]$. Figure 7 shows an example of the results that can be attained within secluded dark matter models. The figure shows limits on the mediator decay length as a function of the the annihilation rate in the Sun (left plot) and limits on the spin-independent dark matter-nucleon cross section as a function of dark matter (right plot) obtained with public data of IceCube and ANTARES [180]. Note that neutrino telescopes, IceCube in particular, provide the best limits on the spin-independent dark matter-nucleon cross section for dark matter masses above a few $100 \mathrm{GeV}$ for the model described in the reference, improving even over direct search experiments like LUX, shown in the figure as the black dot-dashed line.
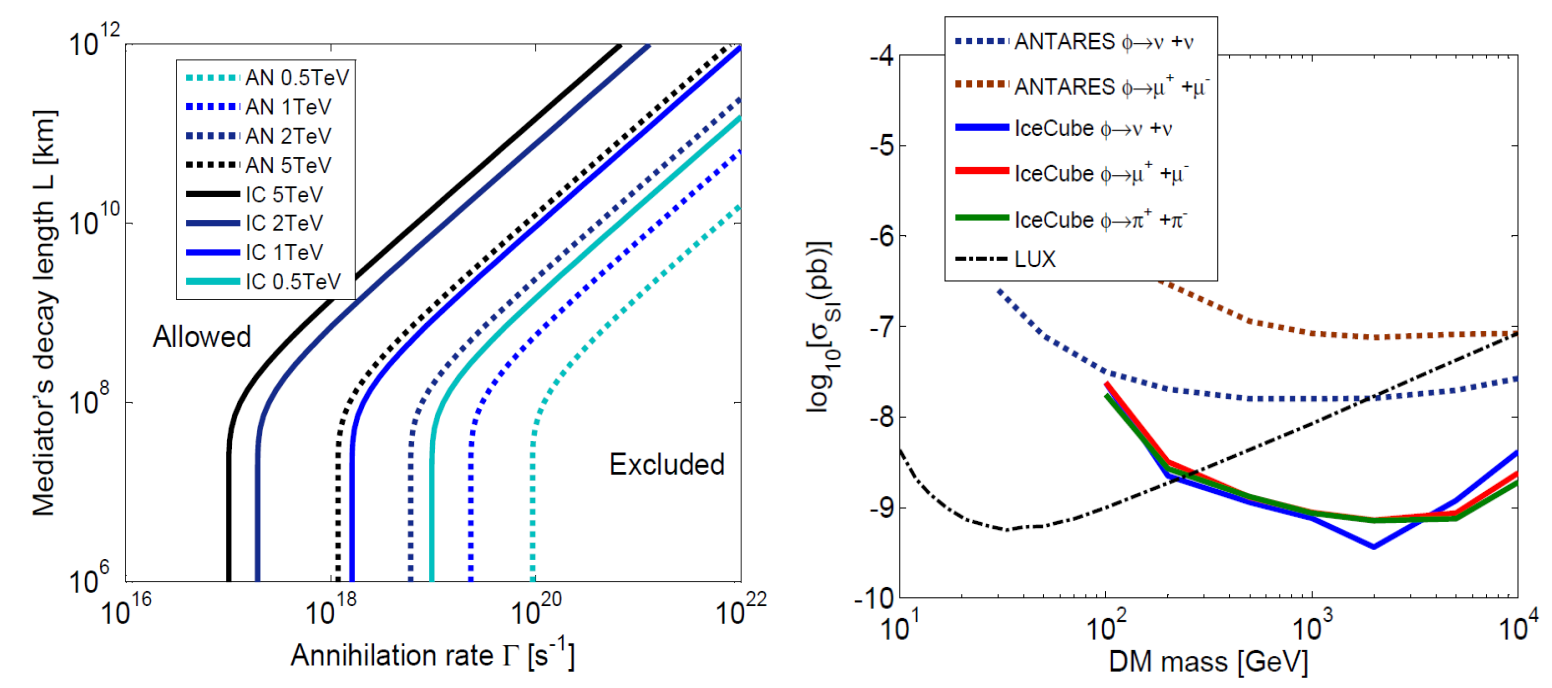

Figure 7. (Left) The $90 \%$ confidence level limits on the mediator decay length as a function of the the annihilation rate in the Sun for a several dark matter masses. The mediator is assumed to decay $100 \%$ to neutrinos. Regions to the right of the curves are disfavoured. See the reference below for other decay modes. (Right) The 90\% confidence level limits on the spin-independent dark matter-nucleon cross section as a function of dark matter mass for different mediator decay channels, assuming a decay length of $2.8 \times 10^{7} \mathrm{~km}$. Figures from [180]. Copyright IOP Publishing Ltd. and Sissa Medialab. Reproduced by permission of IOP Publishing. All rights reserved.

The Earth presents somewhat a different case, since the processes of capture of dark mater and annihilation in its core can not be assumed to have reached equilibrium. Additionally, the most abundant isotopes of the main components of the Earth inner core, mantle and crust, ${ }^{56} \mathrm{Fe},{ }^{28} \mathrm{Si}$ and ${ }^{16} \mathrm{O}$ [182], are spin-0 nuclei, so capture in the Earth is a good candidate to probe the $\sigma_{\mathrm{SI}}$ component of the dark matter-nucleon cross section. From the experimental side, the mass of these isotopes produces mass resonances in the capture rate, so neutrino searches for dark matter annihilations from the centre of the Earth are going to be more sensitive to dark matter masses similar to the masses of those isotopes than to other masses. This is reflected in the shape of the limits obtained by IceCube, ANTARES and Super-Kamiokande, shown in Figure 8. The figure shows current limits on the spin-independent dark matter-nucleon cross section as a function of dark matter mass [183]. 


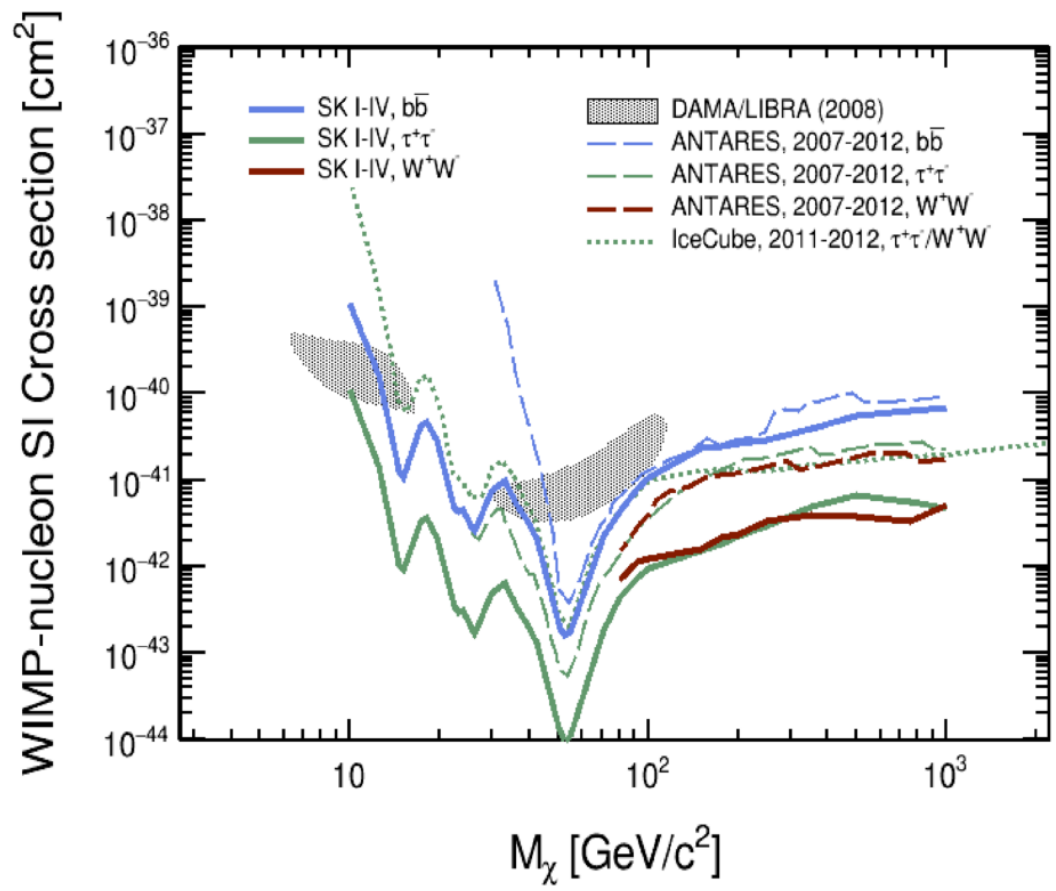

Figure 8. The $90 \%$ confidence level upper limit on the spin-independent dark matter-nucleon cross section from annihilations in the Earth core for different annihilation channels. The shaded areas represent the best fit if one interprets the results from DAMA/LIBRA as indeed originating from dark matter interactions. Figure from [183]. See references therein for details mentioned in the inset caption.

However, perhaps of special interest is the recent discovery of a high-energy cosmic neutrino flux by IceCube [184-186], since it opens new possibilities for dark matter scenarios, specifically for annihilations or decays of very heavy dark matter, $O(\mathrm{PeV})$, that is impossible to probe in accelerators. These are called top-down scenarios, where high energy neutrinos are produced from the decay of heavy relic particles, as opposed to bottom-up scenarios where cosmic neutrinos are expected to be produced from the decay of accelerated protons in a source or its surroundings. Additionally, extremely heavy (and extremely light) dark matter scenarios are becoming of interest since classic WIMP models have been disfavoured by accelerator results and the persistent negative results of direct searches. Indeed even the first two PeV cosmic neutrinos detected by IceCube were promptly interpreted within the framework of $\mathrm{PeV}$ mass decaying dark matter candidates (R-parity violating gravitinos, extra dimension fermions or hidden sector gauge bosons) [187]. Decay of such heavy particles into neutrinos would produce a monochromatic line, compatible with the IceCube events at the time. With currently an excess of neutrinos well beyond $7 \sigma$ over the atmospheric neutrino background above $60 \mathrm{TeV}$, and no sources identified except for the blazar TXS 0506+056 [188,189], the interpretation of the IceCube excess as a signature of dark matter has spurred a lot of activity (e.g., [190-201] among others). The most general approach is to assume a two-component flux where a fraction of the IceCube neutrino flux is assumed to originate from unidentified astrophysical sources, and a fraction of the flux comming from heavy dark matter decays. The low statistics of the IceCube data above $1 \mathrm{PeV}$ do not allow for the moment to establish if the flux presents a cut-off or not. A sharp cut-off in the neutrino energy spectrum would favour the dark matter interpretation. Note that dark matter decays would produce also a flux of high energy photons that can be recycled to lower energies through pair production and inverse Compton scattering on ambient radiation. So any model that explains the IceCube cosmic flux from heavy dark matter decay must be consistent with measurements of the gamma-ray sky, as analyses like $[202,203]$ show. Or, vice versa, results from gamma-ray measurements can constrain the decaying dark matter interpretation of neutrino and cosmic ray results within some dark matter models. Current experimental results on the lifetime of heavy dark matter from IceCube [113], compared with limits 
from HAWC and Fermi-LAT, are shown in Figure 9. The IceCube result assumes a two-component fit, with the astrophysical component following a single power law. Lifetimes below $10^{27}-10^{29} \mathrm{~s}$ are disfavoured at $90 \% \mathrm{CL}$, depending on the candidate mass.

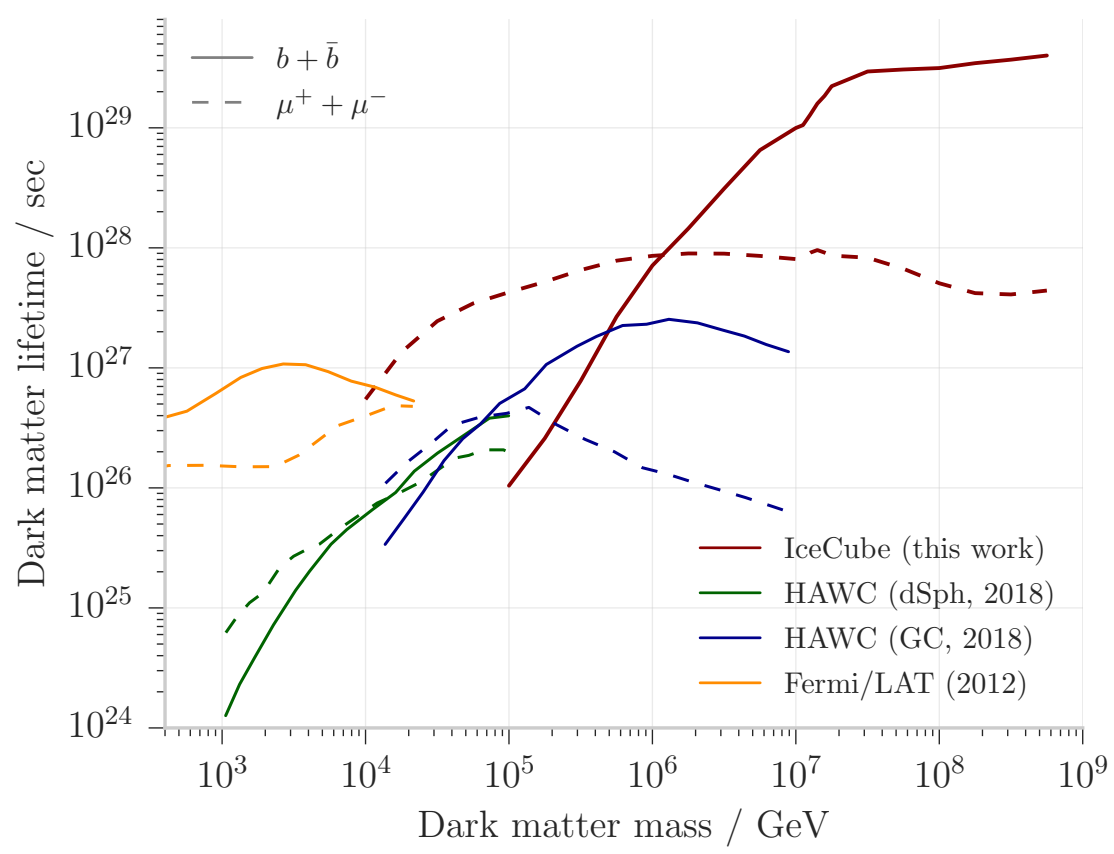

Figure 9. Comparison of IceCube lower lifetime limits on heavy dark matter with results obtained by HAWC (Dwarf Spheroidal Galaxies and Galactic Halo/Centre) and Fermi/LAT available at the time. Figure reproduced with permission from [113]. References to Fermi and HAWC data therein.

The existence of a high-energy cosmic neutrino flux can be used to probe conventional (i.e., not extremely heavy, and even very low mass) dark matter in another, neat, way. Astrophysical neutrinos reach the Earth from far away, traversing dark halos of galaxies in their way, including the Milky Way. Interactions of these neutrinos with the dark matter could reduce the expected neutrino flux from specific directions, providing a unique way of probing the neutrino coupling to the dark sector $[193,194]$. While strongly model-dependent, these approaches can shed light on specific aspects of neutrino-dark matter interactions. An example of the type of results that can be obtained with these analyses is illustrated in Figure 10. The plot shows the result of a search for a depletion of cosmic neutrinos from the direction of the Galactic Centre due to neutrino-dark matter interactions. The results are cast in terms of the mass of a vector-like mediator of the interaction and the dark matter mass, as well as the logarithm of the coupling strength of the interaction (color code). Note that this method is sensitive to very low mass dark matter candidates, on the $\mathrm{MeV}-\mathrm{GeV}$ range, and therefore complementary to other analyses based on the cosmic neutrino flux. The plot also shows that neutrino telescopes have access to an area of the parameter space, typically high mediator masses and high coupling constants, where limits from cosmological arguments based on analyses of the cosmic microwave background do not reach. 


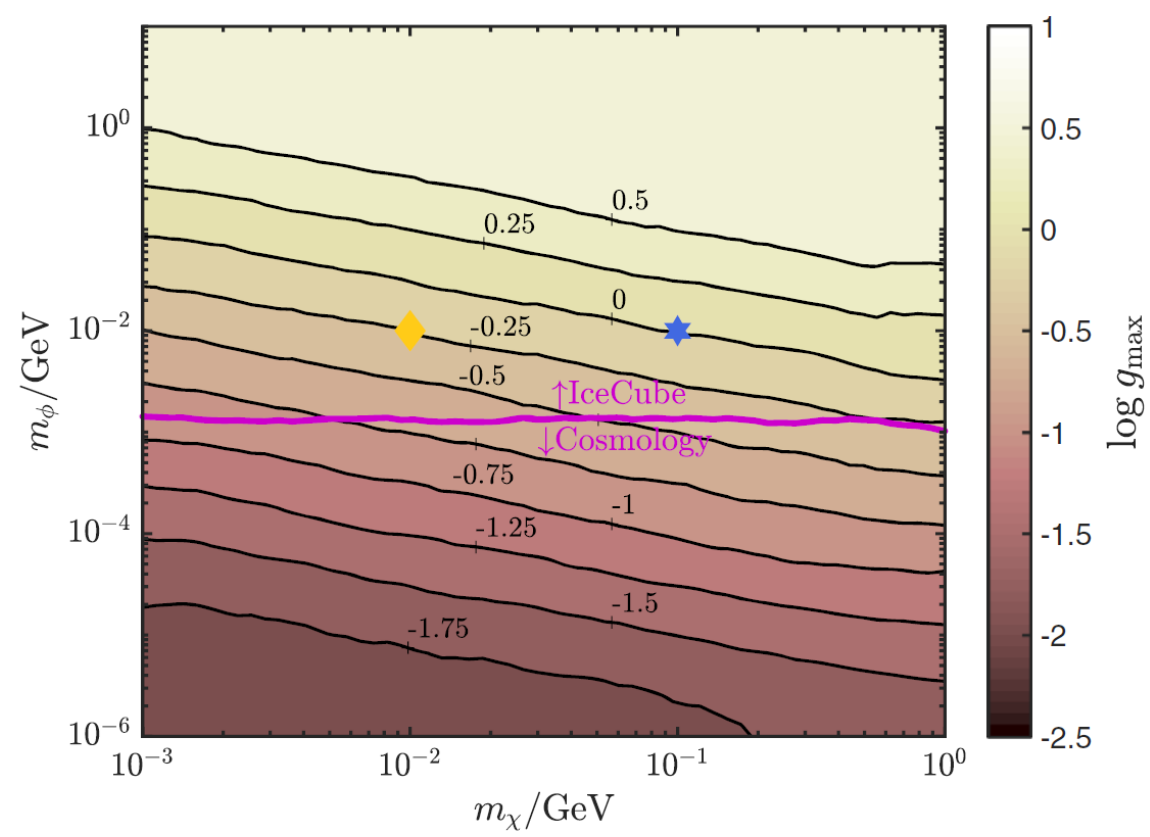

Figure 10. Maximum allowed value (color code) of the (log of the) coupling $g$ between neutrinos and dark matter, as a function of dark matter mass, $m_{\chi}$, when the interaction is mediated by a new vector boson of mass $m_{\phi}$. The horizontal magenta line shows the division between the parameter space regions where a neutrino telescope like IceCube can set stronger limits than analyses based on the power spectrum of the cosmic microwave background, or vice versa. Reprinted with permission from [194]. Copyright 2020 by the American Physical Society.

\subsection{Cosmic-Rays}

Cosmic-rays (protons, electrons or light nuclei and their antiparticles) can also be produced in the hadronization of $q \bar{q}$ or $W^{+} W^{-}$pairs from dark matter annihilations or decays. However, using cosmic-rays as probes of dark matter poses challenges as well. In order to have access to particle identification, charge separation and energy measurement one must deploy real particle physics detectors in space, ideally with calorimetry and a magnetic field for charged-particle tracking capabilities, a technical challenge by itself. However, AMS [204], DAMPE [205] or CALET [206] have demonstrated the feasibility of this type of detectors and produced impressive physics results. The limitations of using cosmic-rays as dark matter probes arise, though, from fundamental physics. Cosmic-rays are charged particles (neutrons being too short lived to be of relevance) and propagation through the Galaxy can randomize their arrival directions and prevent accurate pointing to a given object. Additionally, cosmic-rays can also lose energy through inverse Compton scattering and synchrotron radiation during propagation, so their energy spectrum at Earth might differ from that where dark matter annihilations take place. However, the detection of antimatter (antiprotons, positrons or light antinuclei) still provides a good handle in dark matter searches since antimatter from astrophysical sources is relatively rare at the Earth. Due to its high probability of annihilation with normal matter, any antimatter source needs to be relatively close to the Earth. However, this covers the Galactic Centre and Halo, so there are plenty of predictions of what a detection of an excess of antiprotons or positrons would mean in terms of dark matter. However, even with antimatter searches one has to deal with background from close-by sources, like pulsars, which can produce an (anti)cosmic-ray flux that can be indiscernible from a dark matter signal. Indeed the positron flux (compared to the electron flux) has been the source of controversy since results from the PAMELA satellite [207-209] and the ATIC [210] balloon experiment showed an excess of positrons at energies above $\sim 10 \mathrm{GeV}$ over the expectation from standard galactic primary cosmic-ray propagation and interactions, including the production of secondary electrons and positrons [211,212]. AMS02 [213,214], 
CALET [215] and DAMPE [216] confirmed the excess and showed that it extends to the TeV region, but with a cutoff at around $1 \mathrm{TeV}$. The shape and normalization of the positron excess have given rise to a wealth of models explaining the excess from dark matter annihilations. Some of these solutions are quite contrived since, for example, the antiproton flux does not show such a pronounced deviation from the expected background (there remains to be seen if there is an antiproton excess at all, see below) and therefore an explanation of the positron excess based on dark matter annihilations should prevent the same dark matter to produce a significant excess of antiprotons. These have been called leptophilic models, e.g., [75,217-220] and they are an example of the challenges posed by trying to explain spectral features by invoking multi-component contributions to the spectrum. A fit will always get better the more free parameters are added to the function to fit, without this reflecting on the physical relevance of the parameters. For anyone willing to wield Occam's razor, there is a simpler, standard astrophysical explanation to a positron excess in the cosmic-ray flux: It can indeed be produced by localized pulsar wind nebulae close enough to the Earth [221-226]. In a pulsar wind nebula, positrons are created by electromagnetic cascading from seed electrons that have been ejected from the rapidly spinning neutron star. This electron and positron "wind" eventually creates a shock when interacting with the older ejecta of the original supernova remnant. A fraction of the positrons might escape this complex system into the interstellar medium and become the galactic positron flux. Pulsars are, thus, antimatter sources in the Galaxy. There are known, close enough pulsars to the Earth that can provide the needed positron flux to explain the measured excess over the vanilla, point source free, background. Additionally, a pure dark matter explanation of the positron excess is also strongly disfavoured by the fact that it should have produced a signal in gamma-rays. The lack of a signal in Fermi-LAT searches for dark matter in dwarf spheroid galaxies have set a limit on the dark matter annihilation cross section into channels that would produce an $e^{+} e^{-}$flux which is incompatible with the measured excess $[227,228]$. Again, very few and very contrived models can escape the strong Fermi-LAT constrains and still be compatible with the measurements of PAMELA, ATIC, AMS, CALET and DAMPE.

We mentioned above antiprotons, and the story with them as a dark matter signal is not so simple as it might have been suggested. Antiprotons are produced in interactions of protons and light nuclei with the interstellar medium. So, given the cosmic-ray flux, the distribution of matter in the Galaxy, the antiproton production cross section and a model for propagation, the standard background of antiprotons can be, in principle, calculated. Antiprotons from dark matter annihilations would be produced as final state hadrons in annihilations to $q \bar{q}$ or $W^{+} W^{-}$pairs, and they would appear as an excess over the standard background [229-231]. Figure 11 shows the result of looking for dark matter annihilations into antiprotons with AMS-02. Limits on the velocity-weighted annihilation cross section as a function of dark matter mass for annihilation into $b \bar{b}$ (left) and $W^{+} W^{-}$(right) are shown, along with the $1 \sigma$ and $2 \sigma$ sensitivity bands.

One current problem is that the uncertainties in the ingredients just mentioned above that enter into the calculation of the standard background are larger than present measurements of the antiproton flux by AMS02. The strongest culprit is the uncertainty on the antiproton production cross section in p-p and p-He collisions, which is just known to about $20 \%$, slightly depending on energy, from recent measurements of NA61 and $\mathrm{LHCb}$ [232]. Additionally, uncertainties in the propagation in the Galaxy also play a determinant role. Antiprotons, as charged particles, diffuse in the galactic magnetic field and a complex transport equation, which must include diffusion, convection and reacceleration, with uncertainties in each term, needs to be solved numerically to estimate the flux at the Earth. While there are recurrent claims of a dark matter signal in cosmic-rays from the global fit industry $[233,234]$, the fact remains that, within current uncertainties on the cosmic-ray propagation and on the elementary production cross sections, the data remain compatible with the expected background from standard processes. The simple fact that different authors performing what in principle is a very similar analysis $[234,235]$ reach contradicting conclusions on the significance of the antiproton excess ( $4.7 \sigma$ versus $2.2 \sigma$ ) points to the effect of the uncertainties involved in these type of calculations, mainly due to the cosmic-ray propagation in the galaxy. 

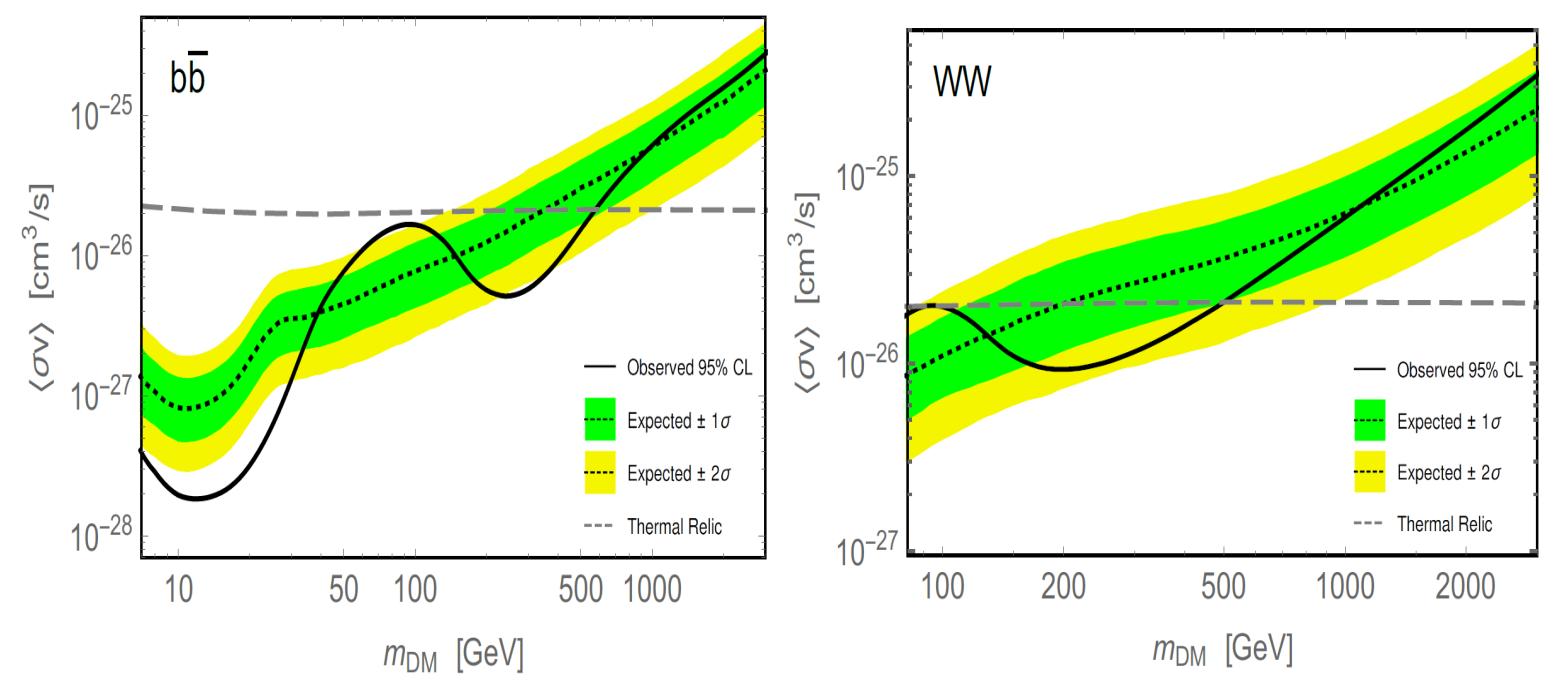

Figure 11. The $95 \%$ confidence level limit on the dark matter annihilation cross section into $b \bar{b}$ (Left) and $W^{+} W^{-}$(Right) as a function of dark matter mass derived from the antiproton and B/C data of AMS-02. Figures from [235]. Copyright IOP Publishing Ltd. and Sissa Medialab. Reproduced by permission of IOP Publishing. All rights reserved.

A way out of this impasse might be the precise measurement of the antideuteron and antihelium fluxes, which should be correlated with the antiproton flux. A comprehensive analysis of the different antimatter cosmic-ray nuclei could help disentangle the standard or exotic origin of these fluxes $[235,236]$.

A very different approach to use cosmic rays as tracers of dark matter is to look for deviations of the expected galactic flux of electrons and protons at the Earth due to interactions with dark matter during their propagation through the galaxy. These interactions will result in a loss of energy of the cosmic rays and a distorted energy spectrum at Earth in comparison with the scenario with no dark matter. This method has been used to set limits on the elastic dark-matter proton and electron cross sections in what has been called "inverse indirect detection" [237]. The argument rests on a similar idea than the one used with neutrinos in [194] and illustrated in Figure 10, but using the galactic cosmic rays as "beam" and the dark matter particles in the galaxy as "target". The method is specially sensitive to low dark matter candidates, well below a GeV, since their number density can then be high enough for several scatterings to occur during the propagation of a cosmic ray, leaving an imprint in their spectrum. This approach complements direct detection experiments that are not currently sensitive to dark matter masses below $O(\mathrm{GeV})$ due to target threshold effects. On the other hand, the prediction on the expected distortion of the $p$ and $e$ spectra depends on the details of the propagation of these particles through the galaxy, which introduces certain degree of uncertainty. In any case, the analysis performed in [237] obtains very competitive limits on the elastic proton and electron cross section with dark matter for dark matter masses as low as a keV. The results are shown in Figure 12 for protons (left plot) and electrons (right plot). The method can be extended to probe more complex interactions, like inelastic scattering or velocity dependent interactions, but at the expense of introducing further model dependencies. 

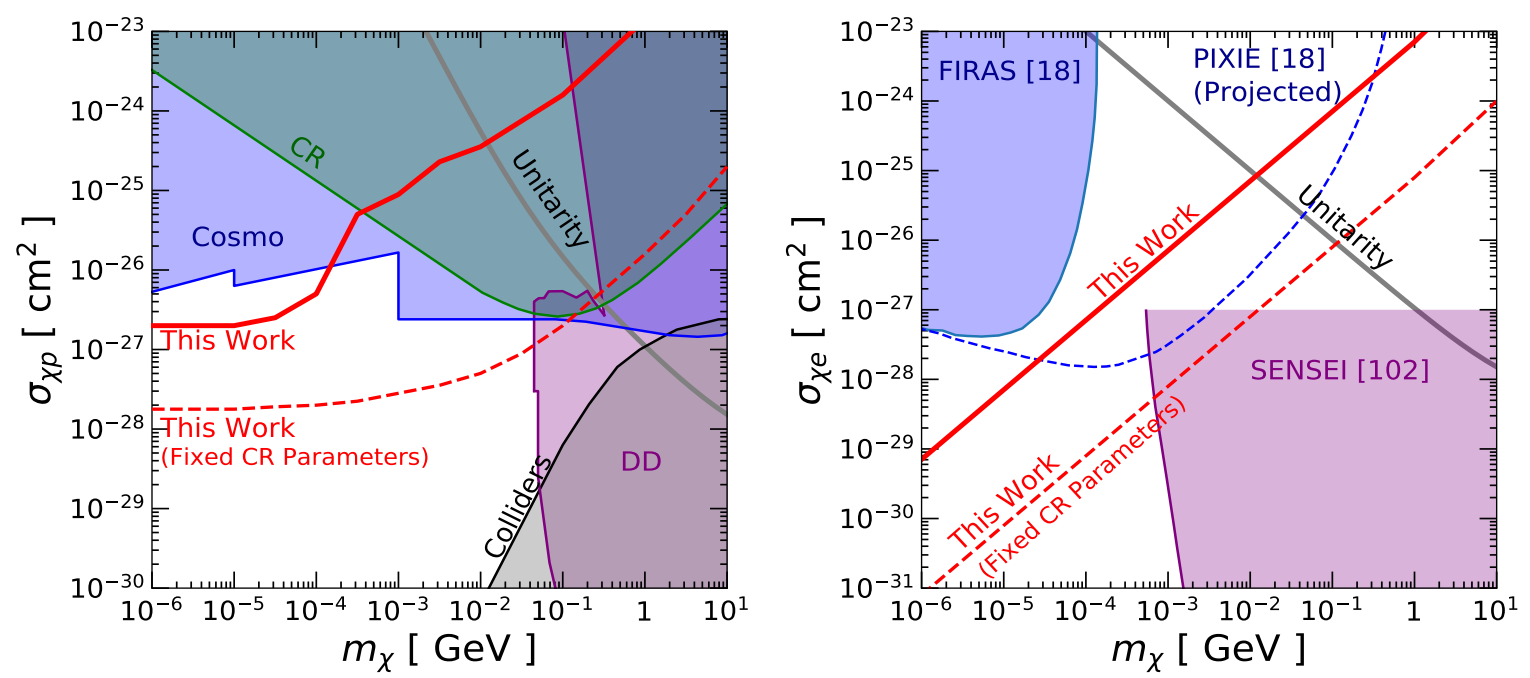

Figure 12. The $5 \sigma$ upper limit (red lines) on the dark matter elastic scattering cross section on protons (left) and electrons (right) obtained by comparing cosmic ray data from AMS-02 and CREAM with the expectation of cosmic ray propagation in the Milky Way including scattering on dark matter. Figures from [237].

\section{Constrains from Cosmology: CMB, BBN and the 21-cm Hydrogen Line}

In this section we summarize the effect of dark matter annihilations and decays in observables related to the evolution of the early universe. This is an independent way to infer the presence and characteristics of dark matter, be it on its imprint on the cosmic microwave background, on the production of primordial light elements through Big-Bang Nucleosynthesis (BBN) or on the 21-cm power spectrum of Hydrogen (see [238] for a review and references therein). The annihilation of dark matter present in the primordial soup injects additional energy in the form of photons, leptons or hadrons into the expanding universe. These will heat, ionize and excite the medium leaving imprints in the expected angular power spectrum that, assuming a given dark matter candidate, can be calculated and compared with observations. Turning the argument around: The consistency of the $\Lambda C D M$ model with observations provides a strong constrain on the characteristics of any new dark matter particle species. These methods have the advantage that they are free from astrophysical uncertainties like halo models of galaxies or dark matter distribution in clusters. They also probe a quite ample range of the history of the universe, from the recombination era $(z=1000)$ to the end of reionization $(z \sim 6)$. Incidentally, a similar line of reasoning can be used to set limits on dark matter annihilations or decays into final states including electromagnetic radiation at later stages in the evolution of the universe. A direct comparison of the predicted photon flux with the extragalactic background glow of the universe (infrared, ultraviolet or even optical) can be used to set constraints to the characteristics of several dark matter candidates [239]. This method is not free from astrophysical uncertainties since it is applied to the older universe, when structures have been formed, and the expected signal depends on how the dark matter, as well as dust and gas, is distributed in galactic halos or the intergalactic medium. The limits obtained are also not as strong as more recent limits mentioned earlier in this review.

Following the evolution of the universe, from higher to lower redshifts, the first effect to consider is the imprint of the energy dumped into the primordial plasma by annihilations or decays of dark matter, and its effect on element nucleosynthesis. Photons, electrons and positrons couple readily to the plasma, but quarks or gluons will hadronize into strongly interacting particles, typically mesons and baryons (and their antiparticles). Short-lived particles (with lifetimes much shorter than the strong interaction rate at the temperature of the surroundings) will decay, reinforcing the energy dumping into the plasma and inducing photo-dissociation of already formed light elements. However, stable particles, like protons (or neutrons for all purposes) will become part of the expanding plasma, ready to participate in nucleosynthesis. Since light-element synthesis depends on the 
baryon-to-photon ratio, additional stable baryons in the plasma due to dark matter annihilations, added to the photo-dissociation mentioned above and pion-exchange interactions that will drive proton-neutron conversion, can change the rate of certain reactions and produce primordial element abundances incompatible with observations. Turning the argument around, the observed abundance of primordial elements can serve to set limits on the amount of dark matter annihilations during the nucleosynthesis era [240-243]. The main uncertainties of this procedure are the uncertainties in measuring the primordial element abundances, a difficult measurement due to the intrinsic contamination of non-primordial elements in any source considered. Measurements of some elements have reached a precision of less than $1 \%\left({ }^{4} \mathrm{He}\right.$ or $\left.\mathrm{D}\right)$, but others like ${ }^{7} \mathrm{Li}$ remain at about $20 \%$ [244]. Even with these caveats, BBN arguments allow to set quite stringent limits on $\left\langle\sigma_{\mathrm{A}} v\right\rangle$ as a function of dark matter mass, as it is shown in the right plot of Figure 13.

Changes in BBN processes will bear on the temperature and polarization power spectra of the $\mathrm{CMB}$. The $\mathrm{CMB}$ conveys detailed information about the last scattering surface and, if dark matter decayed or annihilated in the early universe, the extra energy deposited in the plasma could delay recombination or smooth out the primordial density fluctuations. Such an effect would be visible in the CMB data today [245-247]. However, not all the energy released in the annihilation of two dark matter particles is absorbed into the surrounding medium and it is usual to express results from CMB analyses in terms of an efficiency factor, $f(z)$, which represents the fraction of the annihilation energy that goes into heating and ionizing the surrounding plasma. This factor depends both on redshift and the specific dark matter model through the branching ratios to particles that couple to the plasma, typically photons and electrons and positrons, but it lies in the range 0.001-1 [248]. The redshift dependence of $f(z)$ is less acute than its dependence on the dark matter model, since the universe is evolving fast, and the effects of injecting energy into it are relevant only during a limited $z$ range, typically between 1000 and 600 , so one can approximate $f(z)$ with an effective constant value $f_{\text {eff }}$ which practically only depends on the properties of the dark matter model assumed. Following [248], the energy released per unit volume from dark matter annihilations as the universe expands can be written as

$$
\frac{d E}{d t d V}(z)=2 g^{2} \rho_{\text {crit }}^{2} c^{2} \Omega_{c}^{2}(1+z)^{6} f_{\mathrm{eff}} \frac{\left\langle\sigma_{\mathrm{A}} v\right\rangle}{m_{\mathrm{DM}}}
$$

where $\rho_{\text {crit }}$ the critical density of the universe today, $m_{\mathrm{DM}}$ is the mass of the dark matter particle, and $\left\langle\sigma_{\mathrm{A}} v\right\rangle$ is the thermally-averaged annihilation cross-section times the dark matter velocity. While results from $C M B$ observations are sometimes given as a function of $f_{\text {eff }}\left\langle\sigma_{\mathrm{A}} v\right\rangle$, detailed calculations of the effect of annihilations assuming a simple dark matter model can be performed in order to break the degeneracy and express the results as limits to the $\left\langle\sigma_{A} v\right\rangle$ as a function of the dark matter mass. This has the advantage of presenting results which are directly comparable with other indirect search techniques mentioned in this review, while not being more model dependent. On the contrary, rather less model dependent since, as mentioned above, source modeling does not enter here aside from the usual assumptions of $\Lambda \mathrm{CDM}$ cosmology. There is one caveat, though, when comparing limits on $\left\langle\sigma_{\mathrm{A}} v\right\rangle$ from indirect experiments, probing dark matter accumulated in galactic halos, with limits on $\left\langle\sigma_{\mathrm{A}} v\right\rangle$ from CMB. Note that the direct comparison works under the assumption of s-wave annihilations. For higher order contributions or more complex models, with velocity-dependent annihilation cross section for example, the comparison of limits should be taken with care since they probe very different dark matter velocity regimes. A thermalized $100 \mathrm{GeV}$ particle in a $3000 \mathrm{~K}$ plasma (the temperature of the universe at recombination) has a typical velocity of the order of $100 \mathrm{~m} / \mathrm{s}$, while dark matter particles in galactic halos have typical velocities of $200 \mathrm{~km} / \mathrm{s}$. The left plot of Figure 13 shows limits on $\left\langle\sigma_{\mathrm{A}} v\right\rangle$ as a function of dark matter mass, where a detailed calculation of the effects in the CMB anisotropy induced by the injection of photons, electrons, muons and W's from dark matter annihilations in the expanding plasma has been used to calculate $f_{\text {eff }}$ [249-251]. 

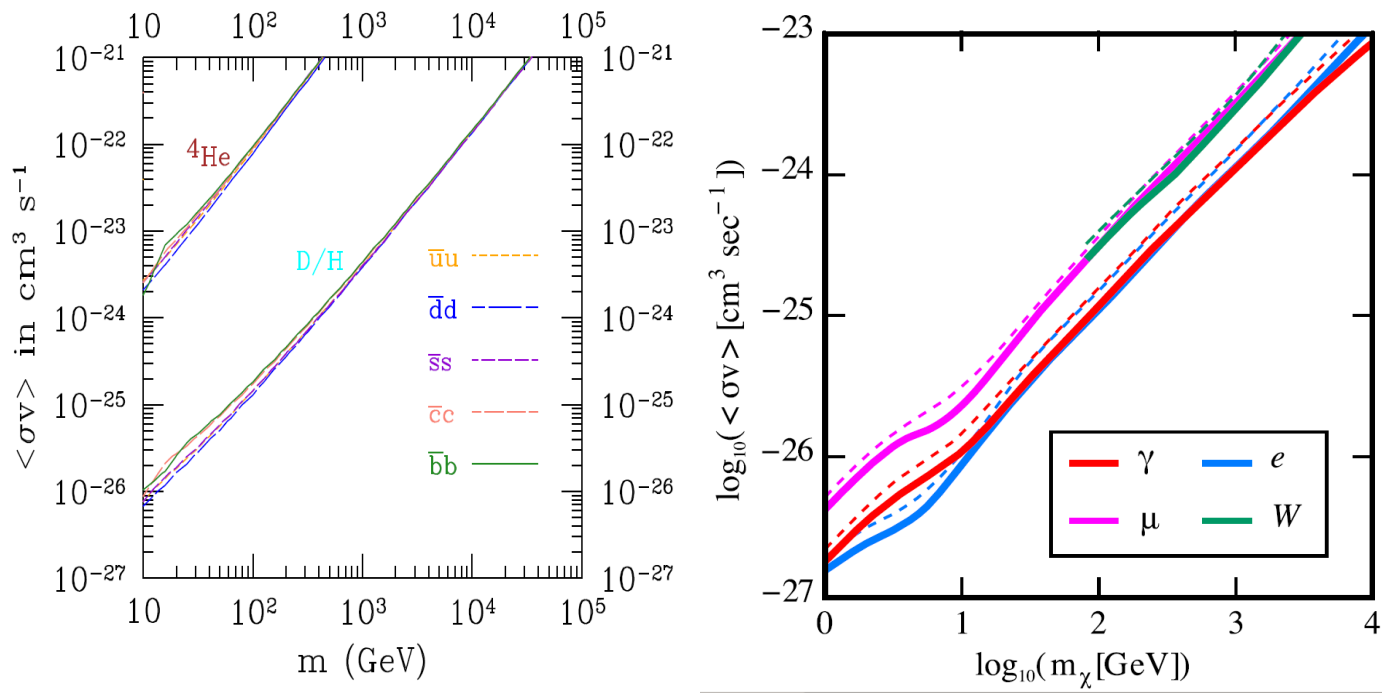

Figure 13. (Left) The 95\% confidence level limits on the velocity-weighted dark matter annihilation cross section as a function of dark matter mass for different annihilation channels, obtained from an analysis on the primordial abundances of light elements from big-bang nucleosynthesis. Figure from [241]. (Right) The 95\% confidence level limits on the velocity-weighted dark matter annihilation cross section as a function of dark matter mass for different annihilation channels, obtained from an analysis of CMB anisotropy data. Figure from [249].

After recombination, the universe enters a phase where primordial atoms have been formed and the ambient radiation does not have enough energy to ionize them. This is called the "Dark Ages", and it lasts until about $\mathrm{z} \sim 200$, when the first large structures start to form by gravitational growth from the primordial fluctuations. As matter collapses into structures it heats up, emitting ultraviolet radiation that can interact with the surrounding gas, specifically inducing a strong absorption at the resonant frequency of the hyper-fine transition between the triplet and the singlet levels of the hydrogen ground state. These two levels are separated by $5.9 \times 10^{-6} \mathrm{eV}$, which corresponds to a wavelength of $21.1 \mathrm{~cm}$ and a frequency of $1420 \mathrm{MHz}$. Today, the redshifted absorption signal appears typically in the 30-200 MHz range and opens the possibility to use radio telescopes as instruments to study the early universe. The $21-\mathrm{cm}$ line has been used to measure rotation curves of galaxies in a complementary way to optical measurements. However, the primordial 21-cm sky follows the matter distribution at the reionization era and provides additional information to CMB cosmology [252]. At about z 6 reionization ends and the whole universe remains ionized until the present epoch. However, up to redshifts about $\mathrm{z} \sim 50$, the $21-\mathrm{cm}$ signal is expected to be negligible. The presence of dark matter annihilations or decays can change this timeline, providing a continuous source of energetic electrons, positrons and gamma-rays that could start the reionization at an earlier time and leave a measurable effect in the 21-cm map [253-257]. Ideally, one could even distinguish the time evolution of dark matter annihilations, which is different from decays. Since the annihilation rate is proportional to $N_{\mathrm{DM}^{\prime}}^{2}$ annihilations can dominate at early times, when the cosmic expansion has not diluted the number density, but they could reignite at a later stage, when dark matter has collapsed into primordial halos, providing a local enhancement of the dark matter density. Since decays are just proportional to $N_{\mathrm{DM}}$, the energy injection into the surrounding medium is uniform with time $[258,259]$. Given the wide energy spectrum of the annihilation/decay products of dark matter (up to the dark matter mass) and the resonance character of ionization, only a small fraction of the injected energy from dark matter can be used for ionization. This limits the dark matter models that can be probed by $21-\mathrm{cm}$ line cosmology to low-mass, typically $O(\mathrm{MeV})$, candidates. Only a tiny fraction of the spectrum of annihilations of heavier candidates is effective for ionization, so the strength of the method decreases rapidly with dark matter mass [260-264]. 
As we showed for the case of the $\mathrm{CMB}$, the energy injected into the intergalactic medium due to dark matter annihilations can be related to the annihilation cross section and candidate mass. There are several ways to express this, as fraction of ionized Hydrogen as a function of redshift, as the temperature of the Hydrogen gas as function of redshift or, what I think gives a more visual connection to the dark matter characteristics, as the rate of energy deposition from annihilations as a function of redshift [265],

$$
\frac{d E}{d t}(z)=\frac{1}{2} N_{\mathrm{DM}, 0}^{2} N_{\mathrm{b}, 0}(1+z)^{3} f_{\mathrm{abs}}(z) \frac{\left\langle\sigma_{\mathrm{A}} v\right\rangle}{m_{\mathrm{DM}}}
$$

where $N_{\mathrm{DM}, 0}$ is the current number density of dark matter particles, $N_{\mathrm{b}, 0}$ is the current baryon number density, $f_{\text {abs }}$ is the fraction of the energy released by the dark matter annihilations that goes into ionization and, as in Equation (6), $m_{\mathrm{DM}}$ is the mass of the dark matter particle and $\left\langle\sigma_{\mathrm{A}} v\right\rangle$ is the thermally-averaged annihilation cross-section times the dark matter velocity. The calculation of the left hand side of Equation (7) is not easy since the injection of energy occurs in a complex and expanding medium, but once a model for the evolution of the intergalactic medium is in place, observations of the 21-cm primordial intensity in the sky (proportional to the injected energy from dark matter annihilations) can be used to constrain $\left\langle\sigma_{\mathrm{A}} v\right\rangle$ as a function of $m_{\mathrm{DM}}$. The main foreground comes from the contribution from later processes, like radio emission from synchrotron processes in our galaxy or from other galaxies and clusters of galaxies, which has to be carefully dealt with.

There are several radio observatories in operation that have the sensitivity to set competitive limits on dark matter annihilations and, indeed, observations from the EDGES telescope [266] have been used to set limits on dark matter characteristics. The EDGES telescope measured the brightness temperature of the $21-\mathrm{cm}$ line relative to the $\mathrm{CMB}$, finding that the absorption profile is consistent with expectations. However, it also found that the relative amplitude of the absorption is more than a factor of two larger than the predictions from standard cosmology at a $3.8 \sigma$ confidence level. Since we are talking of a relative measurement of $T_{21 \mathrm{~cm}}$ with respect to $T_{\mathrm{CMB}}$, the discrepancy can be interpreted either as the primordial gas at $\mathrm{z} \sim 20$ being colder than expected, or the background radiation being hotter than expected $\left(T_{21} \mathrm{~cm}\right.$ and $T_{\mathrm{CMB}}$ denote, respectively, the temperature of the ambient gas and of the $\mathrm{CMB}$ at the end of the Dark Ages). Standard explanations assuming increased radiation from the early stars and/or a slightly different decoupling redshift between matter and radiation can (more or less) accommodate the EDGES measurement, since there are still large uncertainties on the early star formation history of the universe. However, the result from EDGES can also be interpreted in terms of dark matter. Indeed, interactions of dark matter with surrounding baryons or the injection of energy due to dark matter decays or annihilations can change the thermal history of the hydrogen gas and accommodate the observation by EDGES [255,267-269]. The contribution of dark matter annihilation products to heating the background radiation can lead to a suppression of the observed absorption (even erasing it if the energy injection is too large), so the observation of an anomalous absorption level in the 21-cm line sky can be translated into bounds on dark matter annihilations or decays. On the other hand, interactions between dark matter and surrounding baryons can lower the ambient gas temperature, and can explain the observed feature if the dark matter species is light (below a few $\mathrm{GeV}$ ) and its interaction cross section lies above a few $10^{-21} \mathrm{~cm}^{2}$ [269]. Figure 14 shows an example of the constraints obtained from the EDGES observation for two annihilation channels, and assuming that the energy deposited from the annihilations is absorbed by the surrounding plasma with some delay [255]. This needs to be modeled and adds a source of uncertainty in the calculations but it is justified for some annihilation products that can penetrate the gas before fully interacting with it. In other cases, like short-lived particles, a "prompt" energy deposition approximation is more accurate. See [255] for additional limits obtained under the prompt energy deposition approximation. Note that the limits reach lower masses than other indirect searches, but the caveat mentioned above about the different average velocities of dark matter today (as probed by annihilations in the halo of our Galaxy or nearby galaxies) and at higher redshifts (as probed by the 21-cm line) remains when trying to compare results. 


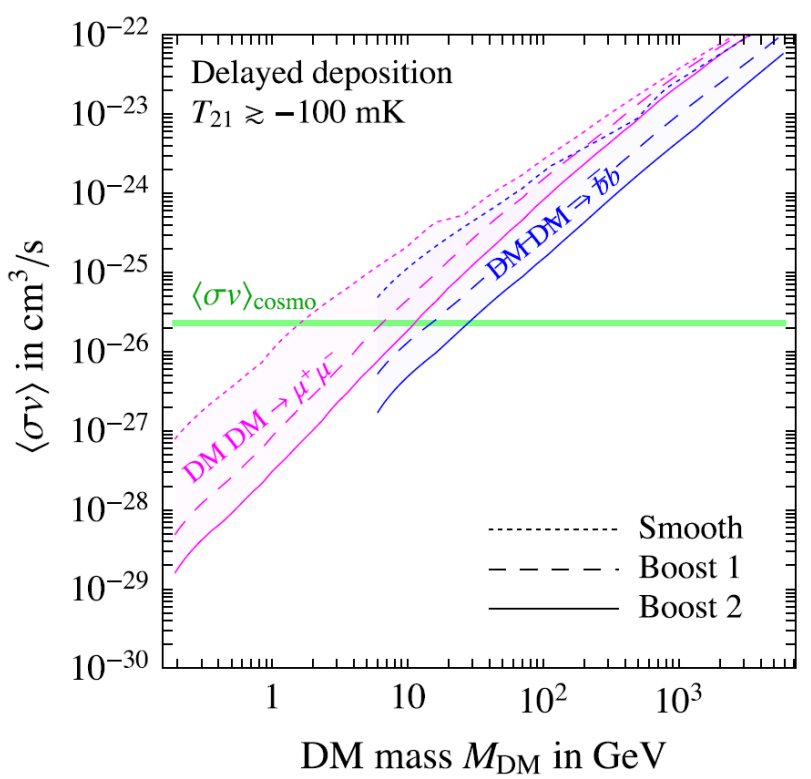

Figure 14. Limits on the velocity-weighted dark matter annihilation cross section as a function of dark matter mass from the absorption observed in the 21-cm spectrum at $z \sim 17$, for two examples of annihilation channels. Figure from [255].

Constrains on the dark matter annihilation cross section can also be obtained by using radio telescopes to target specific sources, in a similar way that it is done with gamma-ray, X-ray and neutrino telescopes. The technique is based on looking for synchrotron radio emission from the charged products (mainly $\mathrm{e}^{-}$and $\mathrm{e}^{+}$) of dark matter annihilations in the halo of a suitable galaxy. The author in [270] used Draco as early as 2002 to set limits on the dark matter velocity averaged self annihilation cross section from VLA observations. More recently, the LOFAR collaboration has carried out a competitive search for dark matter in the dwarf galaxy Canis Venatici [271]. The results of this technique depend on the diffusion of the charged particles once emitted and on the profile of the local magnetic field, two quantities that are not well known, so the results are ineluctably model-dependent. However, with reasonable assumptions on the structure and magnetic field strength from a given source, this method yields competitive results to gamma-ray, cosmic-ray or neutrino dark matter searches from dwarf galaxies as illustrated in Figure 15.

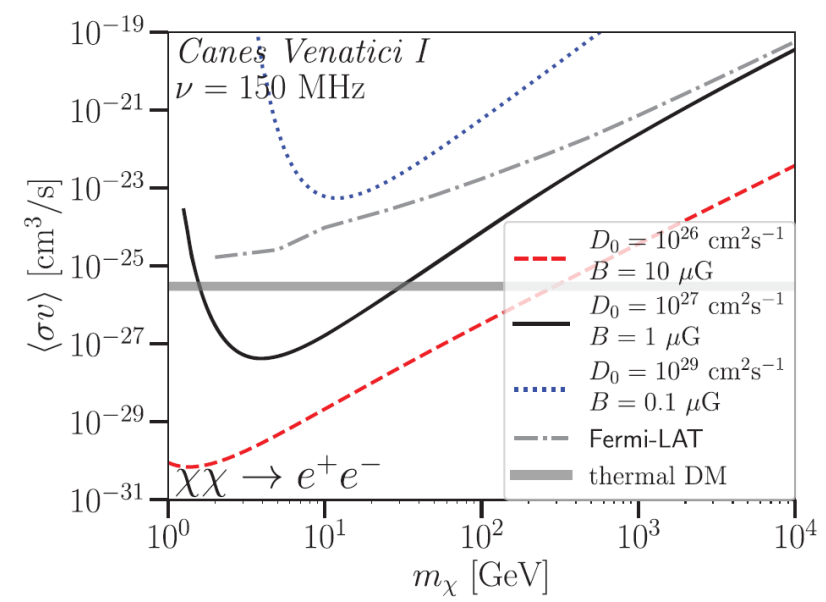

Figure 15. Limits on the velocity-weighted dark matter annihilation cross section as a function of dark matter mass from LOFAR, assuming annihilation into $e^{+} e^{-}$. Results are dependent on the galactic diffusion model used and assumed magnetic field strengths, and results are shown for three different models (blue dotted, black full and red dashed lines). Figure from [271]. See reference for details in the diffusion models used. 


\section{Discussion}

Even that severe constraints from accelerator experiments have closed a big chunk of the supersymmetric parameter space, which was originally a favourite to provide a dark matter candidate with the right mass and interaction strength, the possibilities for particle candidates remain quite wide: Models with very low or very high masses and/or very weak or strong interactions are still viable and escape current accelerator constraints. On the experimental side, the sensitivity of neutrino telescopes, gamma- and X-ray telescopes and cosmic-ray detectors has improved enormously, sometimes by orders of magnitude, in the last decades, but the fact remains that no experiment has reported any positive signal so far. We seem to have past the point where a clear, strong, unambiguous signal will be detected by any given experiment. It seems probable that, rather, any detection of particle dark matter, if that is the solution to our current observational conundrum, will show itself as a slight deviation from the background. Given the uncertainties in the backgrounds of some of the techniques mentioned in this review, it will not suffice that a single experiment detects an anomaly, but that a consistent picture emerges from all the signatures that experimentalists are looking for. I have mentioned Occam's razor a couple of times in this review precisely because, taken individually, the dark matter explanation of any single observation might easily be superseded by an hypothesis using only conventional physics. It is in the correlation between results of different experiments where we should look. Paraphrasing Viktor Hess in his Nobel lecture in 1936: In order to make further progress, particularly in the field of dark matter searches, it will be necessary to apply all our resources and apparatus simultaneously and side-by-side (while the original formulation referred to the "field of cosmic rays" [272]). The aim of this review is to give a glimpse on the signatures looked for in indirect searches for dark matter, accompanying them with examples of recent results and hopefully conveying to the reader that indirect searches for dark matter is a complex and multidisciplinary field, currently driven by observations.

Searches for the products of dark matter annihilations in our galaxy or nearby galaxies with gamma-ray telescopes provide stringent limits to the dark matter annihilation cross section, mainly at relatively low dark matter masses, in the region of a few $\mathrm{GeV}$ to $\sim 100 \mathrm{GeV}$, but they suffer from complex backgrounds. Dwarf spheroid galaxies are a relatively background free sources that are commonly used in these kind of searches. X-ray telescopes extend the mass reach of gamma-ray telescopes to the few $\mathrm{keV}$ region since they can detect softer signatures from final-state radiation of charged decay or annihilation products of low mass candidates. Neutrino telescopes provide a complementary search approach, currently more sensitive to high dark matter masses, $O(100 \mathrm{GeV})$ and above, while searches with charged cosmic-rays suffer from uncertainties from propagation and relevant cross sections, like antiproton production cross section, needed to understand the production mechanisms at the source and as a background. However, they also provide competitive results, comparable to the two other techniques just mentioned. Large radio arrays have recently joined the effort of searching for annihilations of dark matter in dwarf galaxies by looking for anomalous radio emission from synchrotron radiation of $e^{+}$and $e^{-}$produced as a result of dark matter annihilations or decays. There are new astrophysical uncertainties involved in this kind of technique due to the needed knowledge of the magnetic field of the source and the propagation of the electrons in the complex medium that can be a galactic halo. However, results look promising, and they are competitive with the results from gamma-rays, neutrinos and cosmic-rays under certain assumptions of the structure of the source. So these five, let us call them source-based, searches provide really complementary results and, taken together, will really help characterizing any signal.

On top of the source-based searches, early universe cosmology presents an independent way to search for dark matter, based on the effects of dark matter annihilations (or decay) in the evolution of the early universe. Additional energy deposition on the primordial plasma, from the CMB era to the first formation of structures, must not cause a distortion of the large-scale structure of the universe as we see it today. Quite stringent limits on the annihilation or decay characteristics of dark matter can be extracted from such requirement. 
In the big picture of things the question remains: Is the dark sector really so simple as one stable particle species, while the visible sector comprises several fundamental particles and families? Or, even more radically: Is really the solution to the dark matter problem a particle-based one? From a pure experimental point of view, there is currently no evidence for or against either of these options. The particle solution was historically adopted since extensions of the Standard Model of particle physics predict the existence of new particles, and some of them have the characteristics needed of a dark matter candidate (the "WIMP miracle"), and in order to reduce the parameter space, experimental searches are simply interpreted in terms of a single particle solution. The constraining power of the results presented in this review would be lost if several candidates would have to be taken into account. Not least, the hierarchy of the masses, strength of interactions and annihilation branching ratios of the different candidates would be unknown. This poses a limitation from the experimental point of view, but it should not stop theorists from exploring such scenarios [273-279].

As for the second question above, there are proposed ways out from the dark matter conundrum without the need of dark matter. Modified Newtonian Dynamics (MOND) [9], and extensions thereof, is the more completely developed approach so far, but it requires a modification of Newtonian gravity (or General Relativity for that matter), theories that are understood to be universally valid and, therefore, any proposed modification is, at a minimum, philosophically difficult to swallow. MOND really solves the problem it set out to solve: Explaining the flat rotation curves of galaxies taking into account only the visible baryonic matter. It tends to fail, though, when trying to describe complex halos with velocity streams perpendicular to the galactic plane [280], and has serious difficulties with early universe cosmology, the formation of large scale structure and, specifically, the speed of gravitational waves [281,282]. Not that $\Lambda \mathrm{CDM}$ cosmology is free from problems (a singularity at $t=0$ and the need for an ad-hoc cosmological constant for example; not to mention the need for a new type of dark matter itself). It is not the aim to review here the advantages of $\Lambda$ CDM cosmology over alternative theories of gravity or vice-versa, but I would just like to finish by reminding the reader that the lack of any experimental signal so far from dark matter searches really forces us to keep all our options open.

Funding: This research received no external funding.

Conflicts of Interest: The author declares no conflict of interest.

\section{References}

1. Zwicky, F. Die Rotverschiebung von extragalaktischen Nebeln. Helv. Phys. Acta 1933, 6, 110-127.

2. Bosma, A. 21-cm line studies of spiral galaxies. 2. The distribution and kinematics of neutral hydrogen in spiral galaxies of various morphological types. Astron. J. 1981, 86, 1825. [CrossRef]

3. Sofue, Y.; Rubin, V. Rotation curves of spiral galaxies. Ann. Rev. Astron. Astrophys. 2001, 39, 137-174. [CrossRef]

4. Castiblanco, L.; Gannouji, R.; Stahl, C. Large scale structures: From inflation to today: A brief report. arXiv 2019, arXiv:1910.03931.

5. Cyburt, R.H.; Fields, B.D.; Olive, K.A.; Yeh, T.H. Big Bang Nucleosynthesis: 2015. Rev. Mod. Phys. 2016, 88, 015004. [CrossRef]

6. Planelles, S.; Schleicher, D.R.G.; Bykov, A.M. Large-Scale Structure Formation: From the first non-linear objects to massive galaxy clusters. Space Sci. Rev. 2015, 188, 93-139. [CrossRef]

7. Vogelsberger, M.; Marinacci, F.; Torrey, P.; Puchwein, E. Cosmological Simulations of Galaxy Formation. Nat. Rev. Phys. 2020, 2, 42-66. [CrossRef]

8. Ellis, R.S. Gravitational lensing: A unique probe of dark matter and dark energy. Phil. Trans. R. Soc. 2010, 368, 967-987. [CrossRef] [PubMed]

9. Famaey, B.; McGaugh, S. Modified Newtonian Dynamics (MOND): Observational Phenomenology and Relativistic Extensions. Living Rev. Rel. 2012, 15, 10. [CrossRef] [PubMed]

10. Deur, A. An explanation for dark matter and dark energy consistent with the Standard Model of particle physics and General Relativity. Eur. Phys. J. C 2019, 79, 883. [CrossRef] 
11. Christodoulou, D.M.; Kazanas, D. The Case Against Dark Matter and Modified Gravity: Flat Rotation Curves Are a Rigorous Requirement in Rotating Self-Gravitating Newtonian Gaseous Disks. J. Mod. Phys. 2016, 7, 680. [CrossRef]

12. Khoury, J. Alternative to particle dark matter. Phys. Rev. D 2015, 91, 024022. [CrossRef]

13. Steigman, G.; Dasgupta, B.; Beacom, J.F. Precise Relic WIMP Abundance and its Impact on Searches for Dark Matter Annihilation. Phys. Rev. D 2012, 86, 023506. [CrossRef]

14. Blum, K.; Cui, Y.; Kamionkowski, M. An Ultimate Target for Dark Matter Searches. Phys. Rev. D 2015, 92, 023528. [CrossRef]

15. Leane, R.K.; Slatyer, T.R.; Beacom, J.F.; Ng, K.C.Y. GeV-scale thermal WIMPs: Not even slightly ruled out. Phys. Rev. D 2018, 98, 023016. [CrossRef]

16. Baer, H.; Choi, K.Y.; Kim, J.E.; Roszkowski, L. Dark matter production in the early Universe: Beyond the thermal WIMP paradigm. Phys. Rept. 2015, 555, 1. [CrossRef]

17. Schumann, M. Direct Detection of WIMP Dark Matter: Concepts and Status. J. Phys. G 2019, 46, 103003. [CrossRef]

18. Buchmueller, O.; Doglioni, C.; Wang, L.T. Search for dark matter at colliders. Nat. Phys. 2017, 13, $217-223$. [CrossRef]

19. Kahlhoefer, F. Review of LHC Dark Matter Searches. Int. J. Mod. Phys. A 2017, 32, 1730006. [CrossRef]

20. Bondi, M. Searching for light dark matter at fixed target experiments. J. Phys. Conf. Ser. 2020, 1561, 012005. [CrossRef]

21. Sanders, R.H. The Dark Matter Problem: A Historical Perspective; Cambridge University Press: Cambridge, UK, 2010; ISBN 9780521113014.

22. Bertone, G.; Hooper, D. History of dark matter. Rev. Mod. Phys. 2018, 90, 045002. [CrossRef]

23. Profumo, S.; Giani, L.; Piattella, O.F. An Introduction to Particle Dark Matter. Universe 2019, 5, 213. [CrossRef]

24. Feng, J.L. Dark Matter Candidates from Particle Physics and Methods of Detection. Ann. Rev. Astron. Astrophys. 2010, 48, 495-545. [CrossRef]

25. Bertone, G.; Hooper, D.; Silk, J. Particle dark matter: Evidence, candidates and constraints. Phys. Rept. 2005, 405, 279-390. [CrossRef]

26. Gould, A.; Draine, B.T.; Romani, R.W.; Nussinov, S. Neutron Stars: Graveyard of Charged Dark Matter. Phys. Lett. B 1990, 238, 337. [CrossRef]

27. Basdevant, J.L.; Mochkovitch, R.; Rich, J.; Spiro, M.; Vidal-Madjar, A. Is There Room for Charged Dark Matter? Phys. Lett. B 1990, 234, 395. [CrossRef]

28. Noz, J.B.M.; Loeb, A. A small amount of mini-charged dark matter could cool the baryons in the early Universe. Nature 2018, 557, 684.

29. Jungman, G.; Kamionkowski, M.; Griest, K. Supersymmetric dark matter. Phys. Rept. 1996, $267,195$. [CrossRef]

30. Pargner, A. Phenomenology of Axion Dark Matter. Ph.D. Thesis, KIT-Fakultät für Physik des Karlsruher Instituts für Technologie, Karlsruhe, Germany, 2019. [CrossRef]

31. Chung, D.J.H.; Kolb, E.W.; Riotto, A. Superheavy dark matter. Phys. Rev. D 1998, 59, 023501. [CrossRef]

32. Chung, D.J.H.; Kolb, E.W.; Riotto, A. Nonthermal supermassive dark matter. Phys. Rev. Lett. 1998, 81, 4048. [CrossRef]

33. Palomares-Ruiz, S. Tests of Dark Matter Scenarios with Neutrino Telescopes. In Probing Particle Physics with Neutrino Telescopes; de los Pérez Heros, C., Ed.; World Scientific: Singapore, 2020; pp. 191-266.

34. Bauer, M.; Plehn, T. Yet Another Introduction to Dark Matter: The Particle Physics Approach. Lect. Notes Phys. 2019, 959. [CrossRef]

35. Khlopov, M.Y. Probes for Dark Matter Physics. Int. J. Mod. Phys. D 2018, 27, 1841013. [CrossRef]

36. Zhang, Y.; Zhao, Y. Unconventional dark matter models: A brief review. Sci. Bull. 2015, 60, 986. [CrossRef]

37. Salucci, P. The distribution of dark matter in galaxies. Astron. Astrophys. Rev. 2019, 27, 2. [CrossRef]

38. Zavala, J.; Frenk, C.S. Dark matter haloes and subhaloes. Galaxies 2019, 7, 81. [CrossRef]

39. Springel, V.; White, S.D.M.; Jenkins, A.; Frenk, C.S.; Yoshida, N.; Gao, L.; Navarro, J.; Thacker, R.; Croton, D.; Helly, J. Simulating the joint evolution of quasars, galaxies and their large-scale distribution. Nature 2005, 435, 629. [CrossRef]

40. Gunn, J.E. Massive galactic halos. I-Formation and evolution. Astrophys. J. 1977, 218, 592. [CrossRef] 
41. Navarro, J.F.; Frenk, C.S.; White, S.D.M. The Structure of cold dark matter halos. Astrophys. J. 1996, $462,563$. [CrossRef]

42. Einasto, J. On the Construction of a Composite Model for the Galaxy and on the Determination of the System of Galactic Parameters. Tr. Astrofiz. Instituta-Alma-Ata 1965, 5, 87.

43. Salucci, P.; Burkert, A. Dark matter scaling relations. Astrophys. J. Lett. 2000, 537, L9. [CrossRef]

44. Moore, B.; Quinn, T.R.; Governato, F.; Stadel, J.; Lake, G. Cold collapse and the core catastrophe. Mon. Not. R. Astron. Soc. 1999, 310, 1147. [CrossRef]

45. Kravtsov, A.V.; Klypin, A.A.; Bullock, J.S.; Primack, J.R. The Cores of dark matter dominated galaxies: Theory versus observations. Astrophys. J. 1998, 502, 48. [CrossRef]

46. Burkert, A. The Structure of dark matter halos in dwarf galaxies. Astrophys. J. Lett. 1995, 447, L25. [CrossRef]

47. De Blok, W.J.G. The Core-Cusp Problem. Adv. Astron. 2010, 2010, 789293. [CrossRef]

48. Benito, M.; Cuoco, A.; Iocco, F. Handling the Uncertainties in the Galactic Dark Matter Distribution for Particle Dark Matter Searches. JCAP 2019, 1903, 33. [CrossRef]

49. Spergel, D.N.; Steinhardt, P.J. Observational evidence for self-interacting cold dark matter. Phys. Rev. Lett. 2000, 84, 3760. [CrossRef]

50. Rocha, M.; Peter, A.H.G.; Bullock, J.S.; Kaplinghat, M.; Garrison-Kimmel, S.; Onorbe, J.; Moustakas, L.A. Cosmological Simulations with Self-Interacting Dark Matter I: Constant Density Cores and Substructure. Mon. Not. R. Astron. Soc. 2013, 430, 81. [CrossRef]

51. Peter, A.H.G.; Rocha, M.; Bullock, J.S.; Kaplinghat, M. Cosmological Simulations with Self-Interacting Dark Matter II: Halo Shapes vs. Observations. Mon. Not. R. Astron. Soc. 2013, 430, 105. [CrossRef]

52. Vogelsberger, M.; Zavala, J.; Loeb, A. Subhaloes in Self-Interacting Galactic Dark Matter Haloes. Mon. Not. R. Astron. Soc. 2012, 423, 3740. [CrossRef]

53. Bernal, N.; Chu, X.; Kulkarni, S.; Pradler, J. Self-interacting dark matter without prejudice. Phys. Rev. D 2020, 101, 055044. [CrossRef]

54. Salucci, P.; Lapi, A.; Tonini, C.; Gentile, G.; Yegorova, I.; Klein, U. The Universal Rotation Curve of Spiral Galaxies. 2. The Dark Matter Distribution out to the Virial Radius. Mon. Not. R. Astron. Soc. 2007, $378,41$. [CrossRef]

55. Baushev, A.N.; Pilipenko, S.V. The central cusps in dark matter halos: Fact or fiction? Phys. Dark Univ. 2020, 30, 100679. [CrossRef]

56. Cirelli, M.; Corcella, G.; Hektor, A.; Hutsi, G.; Kadastik, M.; Panci, P.; Raidal, M.; Sala, F.; Strumia, A. PPPC 4 DM ID: A Poor Particle Physicist Cookbook for Dark Matter Indirect Detection. JCAP 2011, 3, 51. Erratum in 2012, 1210, E01.

57. Hernquist, L. An Analytical Model for Spherical Galaxies and Bulges. Astrophys. J. 1990, 356, 359. [CrossRef]

58. Taylor, J.E.; Silk, J. The Clumpiness of cold dark matter: Implications for the annihilation signal. Mon. Not. R. Astron. Soc. 2003, 339, 505. [CrossRef]

59. Ando, S.; Ishiyama, T.; Hiroshima, N. Halo Substructure Boosts to the Signatures of Dark Matter Annihilation. Galaxies 2019, 7, 68. [CrossRef]

60. Moliné, Á.; Sánchez-Conde, M.A.; Palomares-Ruiz, S.; Prada, F. Characterization of subhalo structural properties and implications for dark matter annihilation signals. Mon. Not. R. Astron. Soc. 2017, 466, 4974. [CrossRef]

61. Peñarrubia, J. Fluctuations of the gravitational field generated by a random population of extended substructures. Mon. Not. R. Astron. Soc. 2018, 474, 1482. [CrossRef]

62. Weber, M.; de Boer, W. Determination of the Local Dark Matter Density in our Galaxy. Astron. Astrophys. 2010, 509, A25. [CrossRef]

63. Ricotti, M. Dependence of the inner dm profile on the halo mass. Mon. Not. R. Astron. Soc. 2003, $344,1237$. [CrossRef]

64. Cautun, M.; Benitez-Llambay, A.; Deason, A.J.; Frenk, C.S.; Fattahi, A.; Gómez, F.A.; Grand, R.J.J.; Oman, K.A.; Navarro, J.F.; Simpson, C.M. The Milky Way total mass profile as inferred from Gaia DR2. Mon. Not. R. Astron. Soc. 2020, 494, 4291. [CrossRef]

65. Buch, J.; Leung, S.C.J.; Fan, J. Using Gaia DR2 to Constrain Local Dark Matter Density and Thin Dark Disk. JCAP 2019, 1904, 26. [CrossRef]

66. Necib, L.; Lisanti, M.; Belokurov, V. Inferred Evidence for Dark Matter Kinematic Substructure with SDSS-Gaia. Astrophys. J. 2019, 874, 3. [CrossRef] 
67. O'Hare, C.A.J.; Evans, N.W.; McCabe, C.; Myeong, G.; Belokurov, V. Velocity substructure from Gaia and direct searches for dark matter. Phys. Rev. D 2020, 101, 023006. [CrossRef]

68. Banik, N.; Bovy, J.; Bertone, G.; Erkal, D.; de Boer, T.J.L. Evidence of a population of dark subhalos from Gaia and Pan-STARRS observations of the GD-1 stream. arXiv 2019, arXiv:1911.02662.

69. Dekel, A.; Silk, J. The origin of dwarf galaxies, cold dark matter, and biased galaxy formation. Astrophys. J. 1986, 303, 39. [CrossRef]

70. Ogiya, G.; Mori, M. The core-cusp problem in cold dark matter halos and supernova feedback: Effects of Oscillation. Astrophys. J. 2014, 793, 46. [CrossRef]

71. Weinberg, D.H.; Bullock, J.S.; Governato, F.; de Naray, R.K.; Peter, A.H.G. Cold dark matter: Controversies on small scales. Proc. Nat. Acad. Sci. USA 2015, 112, 12249. [CrossRef]

72. Del Popolo, A.; Pace, F. The Cusp/Core problem: Supernovae feedback versus the baryonic clumps and dynamical friction model. Astrophys. Space Sci. 2016, 361, 162. [CrossRef]

73. Gondolo, P.; Gelmini, G. Cosmic abundances of stable particles: Improved analysis. Nucl. Phys. B 1991, 360, 145. [CrossRef]

74. Johnson, C.; Caputo, R.; Karwin, C.; Murgia, S.; Ritz, S.; Shelton, J. Search for gamma-ray emission from p-wave dark matter annihilation in the Galactic Center. Phys. Rev. D 2019, 99, 103007. [CrossRef] [PubMed]

75. Arkani-Hamed, N.; Finkbeiner, D.P.; Slatyer, T.R.; Weiner, N. A Theory of Dark Matter. Phys. Rev. D 2009, 79, 015014. [CrossRef]

76. Feng, J.L.; Kaplinghat, M.; Yu, H.B. Sommerfeld Enhancements for Thermal Relic Dark Matter. Phys. Rev. D 2010, 82, 083525. [CrossRef]

77. Campbell, S.; Dutta, B.; Komatsu, E. Effects of Velocity-Dependent Dark Matter Annihilation on the Energy Spectrum of the Extragalactic Gamma-ray Background. Phys. Rev. D 2010, 82, 095007. [CrossRef]

78. Das, A.; Dasgupta, B. Selection Rule for Enhanced Dark Matter Annihilation. Phys. Rev. Lett. 2017, 118, 251101. [CrossRef]

79. Choi, S.M.; Lee, H.M.; Seo, M.S. Cosmic abundances of SIMP dark matter. JHEP 2017, 4, 154. [CrossRef]

80. Clark, S.J.; Dent, J.B.; Dutta, B.; Strigari, L.E. Indirect detection of the partial p-wave via the s wave in the annihilation cross section of dark matter. Phys. Rev. D 2019, 99, 083003. [CrossRef]

81. Sommerfeld, A. Über die Beugung und Bremsung der Elektronen. Ann. Phys. 1931, 403, 257. [CrossRef]

82. Ullio, P.; Bergström, L.; Edsjö, J.; Lacey, C.G. Cosmological dark matter annihilations into gamma-rays-A closer look. Phys. Rev. D 2002, 66, 123502. [CrossRef]

83. Beacom, J.F.; Bell, N.F.; Mack, G.D. General Upper Bound on the Dark Matter Total Annihilation Cross Section. Phys. Rev. Lett. 2007, 99, 231301. [CrossRef] [PubMed]

84. Acciari, V.A.; Ansoldi, S.; Antonelli, L.A.; Engels, A.A.; Baack, D.; Babić, A.; Banerjee, B.; Barres de Almeida, U.; Barrio, J.A.; Becerra González, J.; et al. A search for dark matter in Triangulum II with the MAGIC telescopes. Phys. Dark Univ. 2020, 28, 100529. [CrossRef]

85. Acciari, V.A.; Ansoldi, S.; Antonelli, L.A.; Engels, A.A.; Arcaro, C.; Baack, D.; Babić, A.; Banerjee, B.; Barres de Almeida, U.; Barrio, J.A.; et al. Constraining Dark Matter lifetime with a deep gamma-ray survey of the Perseus Galaxy Cluster with MAGIC. Phys. Dark Univ. 2018, 22, 38. [CrossRef]

86. Giammaria, P.; Aleksić, J.; Lombardi, S.; Maggio, C.; Palacio, J.; Rico, J.; Vanzo, G.; Vazquez Acosta, M.; MAGIC Collaboration. Latest results on searches for dark matter signatures in galactic and extragalactic selected targets by the MAGIC Telescopes. J. Phys. Conf. Ser. 2016, 718, 042024. [CrossRef]

87. Rinchiuso, L.; Moulin, E.; Armand, C.; Poireau, V.; H.E.S.S. Collaboration. Dark Matter search with H.E.S.S. towards ultra-faint dwarf nearby DES satellites of the Milky Way. In Proceedings of the 36th International Cosmic Ray Conference, Madison, WI, USA, 24 July-1 August 2019.

88. Armand, C.; Moulin, E.; Poireau, V.; Rinchiuso, L. Dark Matter searches towards the WLM dwarf irregular galaxy with H.E.S.S. In Proceedings of the 36th International Cosmic Ray Conference, Madison, WI, USA, 24 July-1 August 2019.

89. Abdallah, H.; Aharonian, F.; Benkhali, F.A.; Angüner, E.O.; Arakawa, M.; Arcaro, C.; Arrieta, M.; Backes, M.; Barnard, M.; Becherini, Y.; et al. Searches for gamma-ray lines and 'pure WIMP' spectra from Dark Matter annihilations in dwarf galaxies with H.E.S.S. JCAP 2018, 11, 37.

90. Abdallah, H.; Abramowski, A.; Aharonian, F.; Benkhali, F.A.; Akhperjanian, A.G.; Andersson, T.; Angüner, E.O.; Arrieta, M.; Aubert, P.; Backes, M.; et al. H.E.S.S. Limits on Linelike Dark Matter Signatures in the $100 \mathrm{GeV}$ to $2 \mathrm{TeV}$ Energy Range Close to the Galactic Center. Phys. Rev. Lett. 2016, 117, 151302. [CrossRef] 
91. Cantlay, B.K.; Wechakama, M. Constraints on dark matter annihilation with electron spectrum from VERITAS. J. Phys. Conf. Ser. 2019, 1380, 012071. [CrossRef]

92. Archambault, S.; Archer, A.; Benbow, W.; Bird, R.; Bourbeau, E.; Brantseg, T.; Buchovecky, M.; Buckley, J.H.; Bugaev, V.; Byrum, K.; et al. Dark Matter Constraints from a Joint Analysis of Dwarf Spheroidal Galaxy Observations with VERITAS. Phys. Rev. D 2017, 95, 082001. [CrossRef]

93. Boyarsky, A.; Ruchayskiy, O. Probing the nature of dark matter with deep XMM-Newton observations of the dwarf spheroidal galaxies. Astron. Nachr. 2017, 338, 287. [CrossRef]

94. Ruchayskiy, O.; Boyarsky, A.; Iakubovskyi, D.; Bulbul, E.; Eckert, D.; Franse, J.; Malyshev, D.; Markevitch, M.; Neronov, A. Searching for decaying dark matter in deep XMM-Newton observation of the Draco dwarf spheroidal. Mon. Not. R. Astron. Soc. 2016, 460, 1390. [CrossRef]

95. Borriello, E.; Paolillo, M.; Miele, G.; Longo, G.; Owen, R. Constraints on sterile neutrino dark matter from XMM-Newton observation of M33. Mon. Not. R. Astron. Soc. 2012, 425, 1628. [CrossRef]

96. Boyarsky, A.; Iakubovskyi, D.; Ruchayskiy, O.; Savchenko, V. Constraints on decaying Dark Matter from XMM-Newton observations of M31. Mon. Not. R. Astron. Soc. 2008, 387, 1361. [CrossRef]

97. Neronov, A.; Malyshev, D.; Eckert, D. Decaying dark matter search with NuSTAR deep sky observations. Phys. Rev. D 2016, 94, 123504. [CrossRef]

98. Perez, K.; Ng, K.C.Y.; Beacom, J.F.; Hersh, C.; Horiuchi, S.; Krivonos, R. Almost closing the $v$ MSM sterile neutrino dark matter window with NuSTAR. Phys. Rev. D 2017, 95, 123002. [CrossRef]

99. Roach, B.M.; Ng, K.C.Y.; Perez, K.; Beacom, J.F.; Horiuchi, S.; Krivonos, R.; Wik, D.R. NuSTAR Tests of Sterile-Neutrino Dark Matter: New Galactic Bulge Observations and Combined Impact. Phys. Rev. D 2020, 101, 103011. [CrossRef]

100. Sekiya, N.; Yamasaki, N.Y.; Mitsuda, K. A Search for a keV Signature of Radiatively Decaying Dark Matter with Suzaku XIS Observations of the X-ray Diffuse Background. Publ. Astron. Soc. Jap. 2015, 68, SP1. [CrossRef]

101. Tamura, T.; Iizuka, R.; Maeda, Y.; Mitsuda, K.; Yamasaki, N.Y. An X-ray Spectroscopic Search for Dark Matter in the Perseus Cluster with Suzaku. Publ. Astron. Soc. Jap. 2015, 67, 23. [CrossRef]

102. Urban, O.; Werner, N.; Allen, S.W.; Simionescu, A.; Kaastra, J.S.; Strigari, L.E. A Suzaku Search for Dark Matter Emission Lines in the X-ray Brightest Galaxy Clusters. Mon. Not. R. Astron. Soc. 2015, 451, 2447. [CrossRef]

103. Kusenko, A.; Loewenstein, M.; Yanagida, T.T. Moduli dark matter and the search for its decay line using Suzaku X-ray telescope. Phys. Rev. D 2013, 87, 043508. [CrossRef]

104. Cadena, S.H.; Franco, J.S.; Molina, R.A.; Gammaldi, V.; Karukes, E.; Salucci, P. Constraints on cross-section and lifetime of dark matter with HAWC Observations of dwarf Irregular galaxies. In Proceedings of the 36th International Cosmic Ray Conference, Madison, WI, USA, 24 July-1 August 2019.

105. Abeysekara, A.U.; Albert, A.; Alfaro, R.; Alvarez, C.; Arceo, R.; Arteaga-Velázquez, J.C.; Avila Rojas, D.; Ayala Solares, H.A.; Becerril, A.; Belmont-Moreno, E.; et al. A Search for Dark Matter in the Galactic Halo with HAWC. JCAP 2018, 1802, 49. [CrossRef]

106. Albert, A.; Alfaro, R.; Alvarez, C.; Arceo, R.; Arteaga-Velázquez, J.C.; Avila Rojas, D.; Ayala Solares, H.A.; Bautista-Elivar, N.; Becerril, A.; Belmont-Moreno, E.; et al. Dark Matter Limits from Dwarf Spheroidal Galaxies with The HAWC Gamma-Ray Observatory. Astrophys. J. 2018, 853, 154. [CrossRef]

107. Abazajian, K.N.; Horiuchi, S.; Kaplinghat, M.; Keeley, R.E.; Macias, O. Strong constraints on thermal relic dark matter from Fermi-LAT observations of the Galactic Center. Phys. Rev. D 2020, 102, 043012. [CrossRef]

108. di Mauro, M.; Hou, X.; Eckner, C.; Zaharijas, G.; Charles, E. Search for $\gamma$-ray emission from dark matter particle interactions from Andromeda and Triangulum Galaxies with the Fermi Large Area Telescope. Phys. Rev. D 2019, 99, 123027. [CrossRef]

109. Albert, A.; Anderson, B.; Bechtol, K.; Drlica-Wagner, A.; Meyer, M.; Sánchez-Conde, M.; Strigari, L.; Wood, M.; Abbott, T.M.C.; Abdalla, F.B.; et al. Searching for Dark Matter Annihilation in Recently Discovered Milky Way Satellites with Fermi-LAT. Astrophys. J. 2017, 834, 110. [CrossRef]

110. Wechakama, M.; Cantlay, B.K. Upper limits on dark matter annihilation with the teraelectronvolt cosmic ray spectrum of electrons and positrons from DAMPE. J. Phys. Conf. Ser. 2019, 1380, 012144. [CrossRef] 
111. Adriani, O.; Akaike, Y.; Asano, K.; Asaoka, Y.; Bagliesi, M.G.; Berti, E.; Berti, E.; Bigongiari, G.; Binns, W.R.; Bonechi, S.; et al. Extended Measurement of the Cosmic-Ray Electron and Positron Spectrum from $11 \mathrm{GeV}$ to $4.8 \mathrm{TeV}$ with the Calorimetric Electron Telescope on the International Space Station. Phys. Rev. Lett. 2018, 120, 261102. [CrossRef]

112. $\mathrm{Xu}, \mathrm{W}$. The Latest Results from AMS on the Searches for Dark Matter. In Proceedings of the 28th International Symposium on Lepton Photon Interactions at High Energies, Guangzhou, China, 7-12 August 2017; Wang, W., Xing, Z.-Z., Eds.; World Scientific: Singapore, 2017; p. 205.

113. Aartsen, M.G.; Abbasi, R.; Abdou, Y.; Ackermann, M.; Adams, J.; Antonio Aguilar Sánchez, J.; Ahlers, M.; Altmann, D.; Auffenberg, J.; Bai, X.; et al. Search for neutrinos from decaying dark matter with IceCube. Eur. Phys. J. C 2018, 78, 831. [CrossRef]

114. Aartsen, M.G.; Abbasi, R.; Abdou, Y.; Ackermann, M.; Adams, J.; Antonio Aguilar Sánchez, J.; Ahlers, M.; Altmann, D.; Auffenberg, J.; Bai, X.; et al. Search for Neutrinos from Dark Matter Self-Annihilations in the center of the Milky Way with 3 years of IceCube/DeepCore. Eur. Phys. J. C 2017, 77, 627. [CrossRef]

115. Aartsen, M.G.; Abbasi, R.; Abdou, Y.; Ackermann, M.; Adams, J.; Antonio Aguilar Sánchez, J.; Ahlers, M.; Altmann, D.; Auffenberg, J.; Bai, X.; et al. Search for annihilating dark matter in the Sun with 3 years of IceCube data. Eur. Phys. J. C 2017, 77, 146. [CrossRef]

116. Albert, A.; André, M.; Anghinolfi, M.; Anton, G.; Ardid, M.; Aubert, J.J.; Aublin, J.; Baret, B.; Basa, S.; Belhorma, B.; et al. Search for dark matter towards the Galactic Centre with 11 years of ANTARES data. Phys. Lett. B 2020, 805, 135439. [CrossRef]

117. Albert, A.; André, M.; Anghinolfi, M.; Anton, G.; Ardid, M.; Aubert, J.J.; Aublin, J.; Baret, B.; Basa, S.; Belhorma, B.; et al. Search for Dark Matter Annihilation in the Earth using the ANTARES Neutrino Telescope. Phys. Dark Univ. 2017, 16, 41. [CrossRef]

118. Adrian-Martinez, S.; Albert, A.; André, M.; Anton, G.; Ardid, M.; Aubert, J.J.; Avgitas, T.; Baret, B.; Barrios-Martí, J.; Basa, S.; et al. Limits on Dark Matter Annihilation in the Sun using the ANTARES Neutrino Telescope. Phys. Lett. B 2016, 759, 69. [CrossRef]

119. Avrorin, A.D.; Avrorin, A.V.; Aynutdinov, V.M.; Bannasch, R.; Belolaptikov, I.A.; Brudanin, V.B.; Budnev, N.M.; Danilchenko, I.A.; Demidov, S.V.; Domogatsky, G.V.; et al. Dark matter constraints from an observation of dSphs and the LMC with the Baikal NT200. J. Exp. Theor. Phys. 2017, 125, 80. [CrossRef]

120. Avrorin, A.D.; Avrorin, A.V.; Aynutdinov, V.M.; Bannasch, R.; Belolaptikov, I.A.; Brudanin, V.B.; Budnev, N.M.; Danilchenko, I.A.; Demidov, S.V.; Domogatsky, G.V.; et al. A search for neutrino signal from dark matter annihilation in the center of the Milky Way with Baikal NT200. Astropart. Phys. 2016, 81, 12. [CrossRef]

121. Avrorin, A.D.; Avrorin, A.V.; Aynutdinov, V.M.; Bannasch, R.; Belolaptikov, I.A.; Brudanin, V.B.; Budnev, N.M.; Danilchenko, I.A.; Demidov, S.V.; Domogatsky, G.V.; et al. Search for neutrino emission from relic dark matter in the Sun with the Baikal NT200 detector. Astropart. Phys. 2015, 62, 12. [CrossRef]

122. Boliev, M.M.; Demidov, S.V.; Mikheyev, S.P.; Suvorova, O.V. Search for muon signal from dark matter annihilations inthe Sun with the Baksan Underground Scintillator Telescope for 24.12 years. JCAP 2013, 9, 19. [CrossRef]

123. Desai, S.; Ashie, Y.; Fukuda, S.; Fukuda, Y.; Ishihara, K.; Itow, Y.; Koshio, Y.; Minamino, A.; Miura, M.; Moriyama, S.; et al. Search for dark matter WIMPs using upward through-going muons in Super-Kamiokande. Phys. Rev. D 2004, 70, 083523. [CrossRef]

124. Abe, K.; Bronner, C.; Haga, Y.; Hayato,, Y.; Ikeda, M.; Imaizumi, S.; Ito, H.; Iyogi, K.; Kameda, J.; Kataoka, Y.; et al. Indirect Search for Dark Matter from the Galactic Center and Halo with the Super-Kamiokande Detector. arXiv 2020, arXiv:2005.05109.

125. Choi, K.; Abe, K.; Haga, Y.; Hayato, Y.; Iyogi, K.; Kameda, J.; Kishimoto, Y.; Miura, M.; Moriyama, S.; Nakahata, M.; et al. Search for neutrinos from annihilation of captured low-mass dark matter particles in the Sun by Super-Kamiokande. Phys. Rev. Lett. 2015, 114, 141301. [CrossRef]

126. Actis, M.; Agnetta, G.; Aharonian, F.; Akhperjanian, A.; Aleksić, J.; Aliu, E.; Allan, D.; Allekotte, I.; Antico, F.; Antonelli, L.A.; et al. Design concepts for the Cherenkov Telescope Array CTA: An advanced facility for ground-based high-energy gamma-ray astronomy. Exper. Astron. 2011, 32, 193. [CrossRef]

127. Aartsen, M.G.; Abbasi, R.; Abdou, Y.; Ackermann, M.; Adams, J.; Antonio Aguilar Sánchez, J.; Ahlers, M.; Altmann, D.; Auffenberg, J.; Bai, X.; et al. IceCube-Gen2: The Window to the Extreme Universe. arXiv 2020, arXiv:2008.04323. 
128. Adrian-Martinez, S.; Albert, A.; André, M.; Anton, G.; Ardid, M.; Aubert, J.J.; Avgitas, T.; Baret, B.; Barrios-Martí, J.; Basa, S.; et al. Letter of intent for KM3NeT 2.0. J. Phys. G 2016, 43, 084001.

129. Avrorin, A.D.; Avrorin, A.V.; Aynutdinov, V.M.; Bannasch, R.; Belolaptikov, I.A.; Brudanin, V.B.; Budnev, N.M.; Danilchenko, I.A.; Demidov, S.V.; Domogatsky, G.V.; et al. BAIKAL-GVD: The New-Generation Neutrino Telescope in Lake Baikal. Bull. Russ. Acad. Sci. Phys. 2019, 83, 921. [CrossRef]

130. Abe, K.; Abe, T.; Aihara, H.; Fukuda, Y.; Hayato, Y.; Huang, K.; Ichikawa, A.K.; Ikeda, M.; Inoue, K.; Ishino, H.; et al. Letter of Intent: The Hyper-Kamiokande Experiment-Detector Design and Physics Potential. arXiv 2011, arXiv:1109.3262.

131. Oakes, L.; Oakes, L.; Armand, C.; Charles, E.; Di Mauro, M.; Giuri, C.; Harding, J.P.; Kerszberg, D.; Miener, T.; Moulin, E.; et al. Combined Dark Matter searches towards dwarf spheroidal galaxies with Fermi-LAT, HAWC, HESS, MAGIC and VERITAS. In Proceedings of the 36th International Cosmic Ray Conference, Madison, WI, USA, 24 July-1 August 2019.

132. Hunter, S.D.; Bertsch, D.L.; Catelli, J.R.; Dame, T.M.; Digel, S.W.; Dingus, B.L.; Esposito, J.A.; Fichtel, C.E.; Hartman, R.C.; Kanbach, G.; et al. EGRET observations of the diffuse gamma-ray emission from the galactic plane. Astrophys. J. 1997, 481, 205. [CrossRef]

133. Goodenough, L.; Hooper, D. Possible Evidence for Dark Matter Annihilation in the Inner Milky Way from the Fermi Gamma Ray Space Telescope. arXiv 2009, arXiv:0910.2998.

134. Calore, F.; Cholis, I.; Weniger, C. Background Model Systematics for the Fermi GeV Excess. JCAP 2015, 1503, 38. [CrossRef]

135. Aartsen, M.G.; Abbasi, R.; Abdou, Y.; Ackermann, M.; Adams, J.; Antonio Aguilar Sánchez, J.; Ahlers, M.; Altmann, D.; Auffenberg, J.; Bai, X.; et al. The Fermi Galactic Center GeV Excess and Implications for Dark Matter. Astrophys. J. 2017, 840, 43.

136. Buschmann, M.; Rodd, N.L.; Safdi, B.R.; Chang, L.J.; Mishra-Sharma, S.; Lisanti, M.; Macias, O. Foreground Mismodeling and the Point Source Explanation of the Fermi Galactic Center Excess. Phys. Rev. D 2020, 102, 023023. [CrossRef]

137. Zhong, Y.M.; McDermott, S.D.; Cholis, I.; Fox, P.J. Testing the Sensitivity of the Galactic Center Excess to the Point Source Mask. Phys. Rev. Lett. 2020, 124, 231103. [CrossRef]

138. Bergström, L.; Edsjö, J.; Gustafsson, M.; Salati, P. Is the dark matter interpretation of the EGRET gamma excess compatible with antiproton measurements? JCAP 2006. [CrossRef]

139. Su, M.; Slatyer, T.R.; Finkbeiner, D.P. Giant Gamma-ray Bubbles from Fermi-LAT: AGN Activity or Bipolar Galactic Wind? Astrophys. J. 2010, 724, 1044. [CrossRef]

140. Lee, S.K.; Lisanti, M.; Safdi, B.R.; Slatyer, T.R.; Xue, W. Evidence for Unresolved $\gamma$-Ray Point Sources in the Inner Galaxy. Phys. Rev. Lett. 2016, 116, 051103. [CrossRef]

141. Hooper, D.; Goodenough, L. Dark Matter Annihilation in The Galactic Center as Seen by the Fermi Gamma Ray Space Telescope. Phys. Lett. B 2011, 697, 412. [CrossRef]

142. Hooper, D.; Linden, T. On The Origin Of The Gamma Rays from The Galactic Center. Phys. Rev. D 2011, 84, 123005. [CrossRef]

143. Agrawal, P.; Batell, B.; Fox, P.J.; Harnik, R. WIMPs at the Galactic Center. JCAP 2015. [CrossRef]

144. Calore, F.; Cholis, I.; McCabe, C.; Weniger, C. A Tale of Tails: Dark Matter Interpretations of the Fermi GeV Excess in Light of Background Model Systematics. Phys. Rev. D 2015, 91, 063003. [CrossRef]

145. Achterberg, A.; van Beekveld, M.; Caron, S.; Gómez-Vargas, G.A.; Hendriks, L.; Ruiz de Austri, R. Implications of the Fermi-LAT Pass 8 Galactic Center excess on supersymmetric dark matter. JCAP 2017, 1712, 40. [CrossRef]

146. Essig, R.; Kuflik, E.; McDermott, S.D.; Volansky, T.; Zurek, K.M. Constraining Light Dark Matter with Diffuse X-ray and Gamma-Ray Observations. JHEP 2013, 11, 193. [CrossRef]

147. Jeltema, T.E.; Profumo, S. Dark Matter Detection with Hard X-ray Telescopes. Mon. Not. R. Astron. Soc. 2012, 421, 1215. [CrossRef]

148. Dodelson, S.; Widrow, L.M. Sterile-neutrinos as dark matter. Phys. Rev. Lett. 1994, 72, 17. [CrossRef] [PubMed]

149. Adhikari, R.; Agostini, M.; Ky, N.A.; Araki, T.; Archidiacono, M.; Bahr, M.; Baur, J.; Behrens, J.; Bezrukov, F.; Dev, P.S.B.; et al. A White Paper on keV Sterile Neutrino Dark Matter. JCAP 2017, 2017, 25. [CrossRef]

150. Boyarsky, A.; Drewes, M.; Lasserre, T.; Mertens, S.; Ruchayskiy, O. Sterile neutrino Dark Matter. Prog. Part. Nucl. Phys. 2019, 104, 1. [CrossRef] 
151. Abazajian, K.N. Sterile neutrinos in cosmology. Phys. Rept. 2017, 711-712, 1. [CrossRef]

152. Abazajian, K.N.; Markevitch, M.; Koushiappas, S.M.; Hickox, R.C. Limits on the Radiative Decay of Sterile Neutrino Dark Matter from the Unresolved Cosmic and Soft X-ray Backgrounds. Phys. Rev. D 2007, 75, 063511. [CrossRef]

153. Ng, K.C.Y.; Roach, B.M.; Perez, K.; Beacom, J.F.; Horiuchi, S.; Krivonos, R.; Wik, D.R. New Constraints on Sterile Neutrino Dark Matter from NuSTAR M31 Observations. Phys. Rev. D 2019, 99, 083005. [CrossRef]

154. Boyarsky, A.; Neronov, A.; Ruchayskiy, O.; Shaposhnikov, M. Constraints on sterile neutrino as a dark matter candidate from the diffuse X-ray background. Mon. Not. R. Astron. Soc. 2006, 370, 213. [CrossRef]

155. Asaka, T.; Blanchet, S.; Shaposhnikov, M. The nuMSM, dark matter and neutrino masses. Phys. Lett. B 2005, 631, 151. [CrossRef]

156. Argüelles, C.A.; Diaz, A.; Kheirandish, A.; del Campo, A.O.; Safa, I.; Vincent, A.C. Dark Matter Annihilation to Neutrinos: An Updated, Consistent \& Compelling Compendium of Constraints. arXiv 2019, arXiv:1912.09486.

157. Press, W.H.; Spergel, D.N. Capture by the sun of a galactic population of weakly interacting massive particles. Astrophys. J. 1985, 296, 679. [CrossRef]

158. Krauss, L.M.; Srednicki, M.; Wilczek, F. Solar System Constraints and Signatures for Dark Matter Candidates. Phys. Rev. D 1986, 33, 2079. [CrossRef] [PubMed]

159. Srednicki, M.; Olive, K.A.; Silk, J. High-Energy Neutrinos from the Sun and Cold Dark Matter. Nucl. Phys. B 1987, 279, 804. [CrossRef]

160. Gaisser, T.K.; Steigman, G.; Tilav, S. Limits on Cold Dark Matter Candidates from Deep Underground Detectors. Phys. Rev. D 1986, 34, 2206. [CrossRef] [PubMed]

161. Ritz, S.; Seckel, D. Detailed Neutrino Spectra from Cold Dark Matter Annihilations in the Sun. Nucl. Phys. B 1988, 304, 877. [CrossRef]

162. Gould, A. Resonant Enhancements in WIMP Capture by the Earth. Astrophys. J. 1987, 321, 571. [CrossRef]

163. Gould, A.; Frieman, J.A.; Freese, K. Probing the Earth With Wimps. Phys. Rev. D 1989, 39, 1029. [CrossRef]

164. Steigman, G.; Sarazin, C.L.; Quintana, H.; Faulkner, J. Dynamical interactions and astrophysical effects of stable heavy neutrinos. Astron. J. 1978, 83, 1050. [CrossRef]

165. Spergel, D.N.; Press, W.H. Effect of hypothetical, weakly interacting, massive particles on energy transport in the solar interior. Astrophys. J. 1985, 294, 663. [CrossRef]

166. Engel, J.; Pittel, S.; Vogel, P. Nuclear physics of dark matter detection. Int. J. Mod. Phys. E 1992, 1, 1. [CrossRef]

167. Grevesse, N.; Sauval, A.J. Standard Solar Composition. Space Sci. Rev. 1998, 85, 161. [CrossRef]

168. Catena, R.; Schwabe, B. Form factors for dark matter capture by the Sun in effective theories. JCAP 2015, 1504, 42. [CrossRef]

169. Bernadich, M.C.I.; Pérez de los Heros, C. Limits on Kaluza-Klein dark matter annihilation in the Sun from recent IceCube results. Eur. Phys. J. C 2020, 80, 129.

170. Catena, R.; Hellström, F. New constraints on inelastic dark matter from IceCube. JCAP 2018, 10, 39. [CrossRef]

171. Albuquerque, I.F.M.; Pérez de los Heros, C. Closing the Window on Strongly Interacting Dark Matter with IceCube. Phys. Rev. D 2010, 81, 063510. [CrossRef]

172. Silverwood, H.; Scott, P.; Danninger, M.; Savage, C.; Edsjö, J.; Adams, J.; Brown, A.M.; Hultqvist, K. Sensitivity of IceCube-DeepCore to neutralino dark matter in the MSSM-25. JCAP 2013, 3, 27. [CrossRef]

173. Trotta, R.; Ruiz de Austri, R.; Pérez de los Heros, C. Prospects for dark matter detection with IceCube in the context of the CMSSM. JCAP 2009, 8, 34. [CrossRef]

174. Allahverdi, R.; Bornhauser, S.; Dutta, B.; Richardson-McDaniel, K. Prospects for Indirect Detection of Sneutrino Dark Matter with IceCube. Phys. Rev. D 2009, 80, 055026. [CrossRef]

175. Pospelov, M.; Ritz, A.; Voloshin, M.B. Secluded WIMP Dark Matter. Phys. Lett. B 2008, 662, 53. [CrossRef]

176. Ajello, M.; Atwood, W.B.; Baldini, L.; Barbiellini, G.; Bastieri, D.; Bellazzini, R.; Berenji, B.; Blandford, R.D.; Bloom, E.D.; Bonamente, E.; et al. Constraints on dark matter models from a Fermi-LAT search for high-energy cosmic-ray electrons from the Sun. Phys. Rev. D 2011, 84, 032007. [CrossRef]

177. Profumo, S.; Queiroz, F.S.; Silk, J.; Siqueira, C. Searching for Secluded Dark Matter with H.E.S.S., Fermi-LAT, and Planck. JCAP 2018, 3, 10. [CrossRef]

178. Cuoco, A.; Luque, P.D.; Gargano, F.; Gustafsson, M.; Loparco, F.; Mazziotta, M.; Serini, D. A search for dark matter cosmic-ray electrons and positrons from the Sun with the Fermi Large Area Telescope. Phys. Rev. D 2020, 101, 022002. [CrossRef] 
179. Leane, R.K.; Ng, K.C.Y.; Beacom, J.F. Powerful Solar Signatures of Long-Lived Dark Mediators. Phys. Rev. D 2017, 95, 123016. [CrossRef]

180. Ardid, M.; Felis, I.; Herrero, A.; Martínez-Mora, J. Constraining Secluded Dark Matter models with the public data from the 79-string IceCube search for dark matter in the Sun. JCAP 2017, 4, 10. [CrossRef]

181. Adrián-Martínez, S.; Albert, A.; André, M.; Anton, G.; Ardid, M.; Aubert, J.J.; Avgitas, T.; Baret, B.; Barrios-Martí, J.; Basa, S.; et al. A search for Secluded Dark Matter in the Sun with the ANTARES neutrino telescope. JCAP 2016, 5, 16. [CrossRef]

182. Herndon, J.M. The chemical composition of the interior shells of the Earth. Proc. R. Soc. Lond. Ser. A 1980, $372,1748$.

183. Mijakowski, P. Dark Matter Searches at Super-Kamiokande. J. Phys. Conf. Ser. 2020, 1342, 012075. [CrossRef]

184. Aartsen, M.G.; Abbasi, R.; Abdou, Y.; Ackermann, M.; Adams, J.; Antonio Aguilar Sánchez, J.; Ahlers, M.; Altmann, D.; Auffenberg, J.; Bai, X.; et al. Evidence for High-Energy Extraterrestrial Neutrinos at the IceCube Detector. Science 2013, 342, 1242856.

185. Aartsen, M.G.; Abbasi, R.; Abdou, Y.; Ackermann, M.; Adams, J.; Antonio Aguilar Sánchez, J.; Ahlers, M.; Altmann, D.; Auffenberg, J.; Bai, X.; et al. Observation of High-Energy Astrophysical Neutrinos in Three Years of IceCube Data. Phys. Rev. Lett. 2014, 113, 101101. [CrossRef]

186. Kopper, C. Observation of Astrophysical Neutrinos in Six Years of IceCube Data. In Proceedings of the 35th International Cosmic Ray Conference, Busan, Corea, 12-20 July 2017.

187. Feldstein, B.; Kusenko, A.; Matsumoto, S.; Yanagida, T.T. Neutrinos at IceCube from Heavy Decaying Dark Matter. Phys. Rev. D 2013, 88, 015004. [CrossRef]

188. Aartsen, M.G.; Abbasi, R.; Abdou, Y.; Ackermann, M.; Adams, J.; Antonio Aguilar Sánchez, J.; Ahlers, M.; Altmann, D.; Auffenberg, J.; Bai, X.; et al. Multimessenger observations of a flaring blazar coincident with high-energy neutrino IceCube-170922A. Science 2018, 361, eaat1378.

189. Aartsen, M.G.; Abbasi, R.; Abdou, Y.; Ackermann, M.; Adams, J.; Antonio Aguilar Sánchez, J.; Ahlers, M.; Altmann, D.; Auffenberg, J.; Bai, X.; et al. Neutrino emission from the direction of the blazar TXS 0506+056 prior to the IceCube-170922A alert. Science 2018, 361, 147.

190. Esmaili, A.; Serpico, P.D. Are IceCube neutrinos unveiling PeV-scale decaying dark matter? JCAP 2013, 11, 54. [CrossRef]

191. Bhattacharya, A.; Esmaili, A.; Palomares-Ruiz, S.; Sarcevic, I. Update on decaying and annihilating heavy dark matter with the 6-year IceCube HESE data. JCAP 2019, 5, 51. [CrossRef]

192. Boucenna, S.M.; Chianese, M.; Mangano, G.; Miele, G.; Morisi, S.; Pisanti, O.; Vitagliano, E. Decaying Leptophilic Dark Matter at IceCube. JCAP 2015, 12, 55. [CrossRef]

193. Choi, K.Y.; Kim, J.; Rott, C. Constraining dark matter-neutrino interactions with IceCube-170922A. Phys. Rev. D 2019, 99, 083018. [CrossRef]

194. Argüelles, C.A.; Kheirandish, A.; Vincent, A.C. Imaging Galactic Dark Matter with High-Energy Cosmic Neutrinos. Phys. Rev. Lett. 2017, 119, 201801. [CrossRef]

195. Kachelriess, M.; Kalashev, O.E.; Kuznetsov, M.Y. Heavy decaying dark matter and IceCube high energy neutrinos. Phys. Rev. D 2018, 98, 083016. [CrossRef]

196. Chianese, M.; Miele, G.; Morisi, S. Interpreting IceCube 6-year HESE data as an evidence for hundred TeV decaying Dark Matter. Phys. Lett. B 2017, 773, 591. [CrossRef]

197. Bhattacharya, A.; Gandhi, R.; Gupta, A.; Mukhopadhyay, S. Boosted Dark Matter and its implications for the features in IceCube HESE data. JCAP 2017, 5, 2. [CrossRef]

198. Dev, P.S.B.; Kazanas, D.; Mohapatra, R.N.; Teplitz, V.L.; Zhang, Y. Heavy right-handed neutrino dark matter and PeV neutrinos at IceCube. JCAP 2016, 8, 34. [CrossRef]

199. Murase, K.; Laha, R.; Ando, S.; Ahlers, M. Testing the Dark Matter Scenario for PeV Neutrinos Observed in IceCube. Phys. Rev. Lett. 2015, 115, 071301. [CrossRef] [PubMed]

200. Rott, C.; Kohri, K.; Park, S.C. Superheavy dark matter and IceCube neutrino signals: Bounds on decaying dark matter. Phys. Rev. D 2015, 92, 023529. [CrossRef]

201. Zavala, J. Galactic PeV neutrinos from dark matter annihilation. Phys. Rev. D 2014, 89, 123516. [CrossRef]

202. Murase, K.; Beacom, J.F. Constraining Very Heavy Dark Matter Using Diffuse Backgrounds of Neutrinos and Cascaded Gamma Rays. JCAP 2012, 10, 43. [CrossRef]

203. Cohen, T.; Murase, K.; Rodd, N.L.; Safdi, B.R.; Soreq, Y. $\gamma$-ray Constraints on Decaying Dark Matter and Implications for IceCube. Phys. Rev. Lett. 2017, 119, 021102. [CrossRef] 
204. Lubelsmeyer, K.; von Dratzig, A.S.; Wlochal, M.; Ambrosi, G.; Azzarello, P.; Battiston, R.; Becker, R.; Becker, U.; Bertucci, B.; Bollweg, K.; et al. Upgrade of the Alpha Magnetic Spectrometer (AMS-02) for long term operation on the International Space Station (ISS). Nucl. Instrum. Meth. A 2011, 654, 639. [CrossRef]

205. Fusco, P. The DAMPE experiment and its latest results. J. Phys. Conf. Ser. 2019, 1390, 012063. [CrossRef]

206. Torii, S.; Marrocchesi, P.S.; CALET Collaboration. The CALorimetric Electron Telescope (CALET) on the International Space Station. Adv. Space Res. 2019, 64, 2531. [CrossRef]

207. Adriani, O.; Barbarino, G.C.; Bazilevskaya, G.A.; Bellotti, R.; Boezio, M.; Bogomolov, E.A.; Bonechi, L.; Bongi, M.; Bonvicini, V.; Bottai, S.; et al. An anomalous positron abundance in cosmic rays with energies 1.5-100 GeV. Nature 2009, 458, 607. [CrossRef]

208. Adriani, O.; Barbarino, G.C.; Bazilevskaya, G.A.; Bellotti, R.; Bianco, A.; Boezio, M.; Bogomolov, E.A.; Bongi, M.; Bonvicini, V.; Bottai, S.; et al. Cosmic-Ray Positron Energy Spectrum Measured by PAMELA. Phys. Rev. Lett. 2013, 111, 081102. [CrossRef]

209. Adriani, O.; Barbarino, G.C.; Bazilevskaya, G.A.; Bellotti, R.; Bianco, A.; Boezio, M.; Bogomolov, E.A.; Bongi, M.; Bonvicini, V.; Bottai, S.; et al. Ten years of PAMELA in space. Riv. Nuovo Cim. 2017, 40, 473.

210. Chang, J.; Adams, J.H.; Ahn, H.S.; Bashindzhagyan, G.L.; Christl, M.; Ganel, O.; Guzik, T.G.; Isbert, J.; Kim, K.C.; Kuznetsov, E.N.; et al. An excess of cosmic ray electrons at energies of 300-800 GeV. Nature 2008, 456, 362. [CrossRef] [PubMed]

211. Moskalenko, I.V.; Strong, A.W. Production and propagation of cosmic ray positrons and electrons. Astrophys. J. 1998, 493, 694. [CrossRef]

212. Delahaye, T.; Lavalle, J.; Lineros, R.; Donato, F.; Fornengo, N. Galactic electrons and positrons at the Earth: New estimate of the primary and secondary fluxes. Astron. Astrophys. 2010, 524, A51. [CrossRef]

213. Accardo, L.; Aguilar, M.; Aisa, D.; Alpat, B.; Alvino, A.; Ambrosi, G.; Andeen, K.; Arruda, L.; Attig, N.; Azzarello, P.; et al. High Statistics Measurement of the Positron Fraction in Primary Cosmic Rays of 0.5-500 $\mathrm{GeV}$ with the Alpha Magnetic Spectrometer on the International Space Station. Phys. Rev. Lett. 2014, 113, 121101. [CrossRef]

214. Aguilar, M.; Cavasonza, L.A.; Ambrosi, G.; Arruda, L.; Attig, N.; Azzarello, P.; Bachlechner, A.; Barao, F.; Barrau, A.; Barrin, L.; et al. Towards Understanding the Origin of Cosmic-Ray Positrons. Phys. Rev. Lett. 2019, 122, 041102. [CrossRef]

215. Adriani, O.; Akaike, Y.; Asano, K.; Asaoka, Y.; Bagliesi, M.G.; Berti, E.; Berti, E.; Bigongiari, G.; Binns, W.R.; Bonechi, S.; et al. Energy Spectrum of Cosmic-Ray Electron and Positron from $10 \mathrm{GeV}$ to $3 \mathrm{TeV}$ Observed with the Calorimetric Electron Telescope on the International Space Station. Phys. Rev. Lett. 2017, 119, 181101. [CrossRef]

216. Ambrosi, G.; An, Q.; Asfandiyarov, R.; Azzarello, P.; Bernardini, P.; Bertucci, B.; Cai, M.S.; Chang, J.; Chen, D.Y.; Chen, H.F.; et al. Direct detection of a break in the teraelectronvolt cosmic-ray spectrum of electrons and positrons. Nature 2017, 552, 63.

217. Haba, N.; Kajiyama, Y.; Matsumoto, S.; Okada, H.; Yoshioka, K. Universally Leptophilic Dark Matter from Non-Abelian Discrete Symmetry. Phys. Lett. B 2011, 695, 476. [CrossRef]

218. Cohen, T.; Zurek, K.M. Leptophilic Dark Matter from the Lepton Asymmetry. Phys. Rev. Lett. 2010, 104, 101301. [CrossRef]

219. Ibarra, A.; Ringwald, A.; Tran, D.; Weniger, C. Cosmic Rays from Leptophilic Dark Matter Decay via Kinetic Mixing. JCAP 2009, 8, 17. [CrossRef]

220. Fox, P.J.; Poppitz, E. Leptophilic Dark Matter. Phys. Rev. D 2009, 79, 083528. [CrossRef]

221. Hooper, D.; Blasi, P.; Serpico, P.D. Pulsars as the Sources of High Energy Cosmic Ray Positrons. JCAP 2009, 901, 25. [CrossRef]

222. Profumo, S. Dissecting cosmic-ray electron-positron data with Occam's Razor: The role of known Pulsars. Central Eur. J. Phys. 2011, 10, 1. [CrossRef]

223. Malyshev, D.; Cholis, I.; Gelfand, J. Pulsars versus Dark Matter Interpretation of ATIC/PAMELA. Phys. Rev. D 2009, 80, 063005. [CrossRef]

224. Blasi, P.; Amato, E. Positrons from pulsar winds. In High-Energy Emission from Pulsars and Their Systems, Proceedings of the First Session of the Sant Cugat Forum on Astrophysics, Sant Cugat, Spain, 12-16 April 2010; Torres, D.F., Rea, N., Eds.; Springer: Berlin/Heidelberg, Germany, 2011. [CrossRef]

225. Yin, P.F.; Yu, Z.H.; Yuan, Q.; Bi, X.J. Pulsar interpretation for the AMS-02 result. Phys. Rev. D 2013, 88, 023001. [CrossRef] 
226. Bartels, R.; Krishnamurthy, S.; Weniger, C. Strong support for the millisecond pulsar origin of the Galactic center GeV excess. Phys. Rev. Lett. 2016, 116, 051102. [CrossRef]

227. López, A.; Savage, C.; Spolyar, D.; Adams, D.Q. Fermi/LAT observations of Dwarf Galaxies highly constrain a Dark Matter Interpretation of Excess Positrons seen in AMS-02, HEAT, and PAMELA. JCAP 2016, 1603, 33. [CrossRef]

228. Chan, M.H. Indirect constraints on the dark matter interpretation of excess positrons seen by AMS-02. Phys. Rev. D 2015, 92, 083504. [CrossRef]

229. Bergström, L.; Edsjö, J.; Ullio, P. Cosmic anti-protons as a probe for supersymmetric dark matter? Astrophys. J. 1999, 526, 215. [CrossRef]

230. Donato, F.; Fornengo, N.; Maurin, D.; Salati, P. Antiprotons in cosmic rays from neutralino annihilation. Phys. Rev. D 2004, 69, 063501. [CrossRef]

231. Fornengo, N.; Maccione, L.; Vittino, A. Constraints on particle dark matter from cosmic-ray antiprotons. JCAP 2014, 1404, 3. [CrossRef]

232. Korsmeier, M.; Donato, F.; di Mauro, M. Production cross sections of cosmic antiprotons in the light of new data from the NA61 and LHCb experiments. Phys. Rev. D 2018, 97, 103019. [CrossRef]

233. Cuoco, A.; Heisig, J.; Klamt, L.; Korsmeier, M.; Krämer, M. Scrutinizing the evidence for dark matter in cosmic-ray antiprotons. Phys. Rev. D 2019, 99, 103014. [CrossRef]

234. Cholis, I.; Linden, T.; Hooper, D. A Robust Excess in the Cosmic-Ray Antiproton Spectrum: Implications for Annihilating Dark Matter. Phys. Rev. D 2019, 99, 103026. [CrossRef]

235. Reinert, A.; Winkler, M.W. A Precision Search for WIMPs with Charged Cosmic Rays. JCAP 2018, $1801,55$. [CrossRef]

236. Korsmeier, M.; Donato, F.; Fornengo, N. Prospects to verify a possible dark matter hint in cosmic antiprotons with antideuterons and antihelium. Phys. Rev. D 2018, 97, 103011. [CrossRef]

237. Cappiello, C.V.; Ng, K.C.Y.; Beacom, J.F. Reverse Direct Detection: Cosmic Ray Scattering with Light Dark Matter. Phys. Rev. D 2019, 99, 063004. [CrossRef]

238. Clark, S.J. Constraining Dark Matter through Cosmological Observations. Doctoral Dissertation; Texas A\&M University, 2019. Available online: http://hdl.handle.net/1969.1/186269 (accessed on 28 September 2020).

239. Overduin, J.M.; Wesson, P.S. Dark matter and background light. Phys. Rept. 2004, 402, 267. [CrossRef]

240. Reno, M.; Seckel, D. Primordial Nucleosynthesis: The Effects of Injecting Hadrons. Phys. Rev. D 1988, 37, 3441. [CrossRef]

241. Kawasaki, M.; Kohri, K.; Moroi, T.; Takaesu, Y. Revisiting Big-Bang Nucleosynthesis Constraints on Dark-Matter Annihilation. Phys. Lett. B 2015, 751, 246. [CrossRef]

242. Jedamzik, K. Neutralinos and Big Bang nucleosynthesis. Phys. Rev. D 2004, 70, 083510. [CrossRef]

243. Hisano, J.; Kawasaki, M.; Kohri, K.; Moroi, T.; Nakayama, K. Cosmic Rays from Dark Matter Annihilation and Big-Bang Nucleosynthesis. Phys. Rev. D 2009, 79, 083522. [CrossRef]

244. Fields, B.D.; Olive, K.A.; Yeh, T.H.; Young, C. Big-Bang Nucleosynthesis After Planck. JCAP 2020, 3, 10. [CrossRef]

245. Slatyer, T.R. Indirect dark matter signatures in the cosmic dark ages. I. Generalizing the bound on s-wave dark matter annihilation from Planck results. Phys. Rev. D 2016, 93, 023527. [CrossRef]

246. Slatyer, T.R. Indirect Dark Matter Signatures in the Cosmic Dark Ages II. Ionization, Heating and Photon Production from Arbitrary Energy Injections. Phys. Rev. D 2016, 93, 023521. [CrossRef]

247. Zhang, L.; Chen, X.L.; Lei, Y.A.; Si, Z.G. The impacts of dark matter particle annihilation on recombination and the anisotropies of the cosmic microwave background. Phys. Rev. D 2006, 74, 103519. [CrossRef]

248. Ade, P.; Aghanim, N.; Arnaud, M.; Ashdown, M.; Aumont, J.; Baccigalupi, C.; Banday, A.J.; Barreiro, R.B.; Bartlett, J.G.; Bartolo, N.; et al. Planck 2015 results. XIII. Cosmological parameters. Astron. Astrophys. 2016, 594, A13.

249. Kawasaki, M.; Nakayama, K.; Sekiguchi, T. CMB constraint on dark matter annihilation after Planck 2015. Phys. Lett B 2016, 756, 212. [CrossRef]

250. Kanzaki, T.; Kawasaki, M. Electron and Photon Energy Deposition in Universe. Phys. Rev. D 2008, 78, 103004. [CrossRef]

251. Kanzaki, T.; Kawasaki, M.; Nakayama, K. Effects of Dark Matter Annihilation on the Cosmic Microwave Background. Prog. Theor. Phys. 2010, 123, 853. [CrossRef] 
252. Pritchard, J.R.; Loeb, A. 21-cm cosmology in the 21st century. Rept. Prog. Phys. 2012, 75, 086901. [CrossRef] [PubMed]

253. Evoli, C.; Mesinger, A.; Ferrara, A. Unveiling the nature of dark matter with high redshift $21 \mathrm{~cm}$ line experiments. JCAP 2014, 11, 24. [CrossRef]

254. Belikov, A.V.; Hooper, D. How Dark Matter Reionized The Universe. Phys. Rev. D 2009, 80, 035007. [CrossRef]

255. D'Amico, G.; Panci, P.; Strumia, A. Bounds on Dark Matter annihilations from $21 \mathrm{~cm}$ data. Phys. Rev. Lett. 2018, 121, 011103. [CrossRef]

256. Natarajan, A.; Schwarz, D.J. Dark matter annihilation and its effect on CMB and Hydrogen 21 cm observations. Phys. Rev. D 2009, 80, 043529. [CrossRef]

257. Natarajan, A.; Schwarz, D.J. The effect of early dark matter halos on reionization. Phys. Rev. D 2008, 78, 103524. Erratum in 2010, 81, 089905. [CrossRef]

258. Cumberbatch, D.T.; Lattanzi, M.; Silk, J.; Lattanzi, M.; Silk, J. Signatures of clumpy dark matter in the global $21 \mathrm{~cm}$ Background Signal. Phys. Rev. D 2010, 82, 103508. [CrossRef]

259. Chuzhoy, L. Impact of Dark Matter Annihilation on the High-Redshift Intergalactic Medium. Astrophys. J. Lett. 2008, 679, L65. [CrossRef]

260. Ripamonti, E.; Mapelli, M.; Ferrara, A. Intergalactic medium heating by dark matter. Mon. Not. R. Astron. Soc. 2007, 374, 1067. [CrossRef]

261. Ripamonti, E.; Mapelli, M.; Ferrara, A. The impact of dark matter decays and annihilations on the formation of the first structures. Mon. Not. R. Astron. Soc. 2007, 375, 1399. [CrossRef]

262. Mapelli, M.; Ferrara, A.; Pierpaoli, E. Impact of dark matter decays and annihilations on reionzation. Mon. Not. R. Astron. Soc. 2006, 369, 1719. [CrossRef]

263. Mapelli, M.; Ripamonti, E. Primordial gas heating by dark matter and structure formation. Mem. Soc. Ast. It. 2007, 78, 800 .

264. López-Honorez, L.; Mena, O.; Moliné, Á.; Palomares-Ruiz, S.; Vincent, A.C. The $21 \mathrm{~cm}$ signal and the interplay between dark matter annihilations and astrophysical processes. JCAP 2016, 8, 4. [CrossRef]

265. Valdes, M.; Ferrara, A.; Mapelli, M.; Ripamonti, E. Constraining DM through $21 \mathrm{~cm}$ observations. Mon. Not. R. Astron. Soc. 2007, 377, 245. [CrossRef]

266. Bowman, J.D.; Rogers, A.E.E.; Monsalve, R.A.; Mozdzen, T.J.; Mahesh, N. An absorption profile centred at 78 megahertz in the sky-averaged spectrum. Nature 2018, 555, 67. [CrossRef]

267. Panci, P. 21-cm line Anomaly: A brief Status. Nuovo Cim. C 2020, 42 6, 243.

268. Liu, H.; Slatyer, T.R. Implications of a 21-cm signal for dark matter annihilation and decay. Phys. Rev. D 2018, 98, 023501. [CrossRef]

269. Barkana, R. Possible interaction between baryons and dark-matter particles revealed by the first stars. Nature 2018, 555, 71. [CrossRef]

270. Tyler, C. Particle dark matter constraints from the Draco Dwarf galaxy. Phys. Rev. D 2002, 66, 023509. [CrossRef]

271. Vollmann, M.; Heesen, V.; Shimwell, T.; Hardcastle, M.J.; Brüggen, M.; Sigl, G.; Röttgering, H. Radio constraints on dark matter annihilation in Canes Venatici I with LOFAR. Mon. Not. Roy. Astron. Soc. 2020, 496, 2663. [CrossRef]

272. Hess, V. Unsolved Problems in Physics: Tasks for the Immediate Future in Cosmic Ray Studies. In Nobel Lectures, Physics 1922-1941; Stuart, S., Ed.; Elsevier: Amsterdam, The Netherlands, 1965; ISBN 9781483222486.

273. Yaguna, C.E.; Zapata, Ó. Multi-component scalar dark matter from a $Z_{N}$ symmetry: A systematic analysis. JHEP 2020, 3, 109. [CrossRef]

274. Bélanger, G.; Pukhov, A.; Yaguna, C.E.; Zapata, Ó. The $Z_{5}$ model of two-component dark matter. JHEP 2020, 9, 30. [CrossRef]

275. Elahi, F.; Khatibi, S. Multi-Component Dark Matter in a Non-Abelian Dark Sector. Phys. Rev. D 2019, 100, 015019. [CrossRef]

276. Poulin, A.; Godfrey, S. Multicomponent dark matter from a hidden gauged SU(3). Phys. Rev. D 2019, 99, 076008. [CrossRef]

277. Ahmed, A.; Duch, M.; Grzadkowski, B.; Iglicki, M. Multi-Component Dark Matter: The vector and fermion case. Eur. Phys. J. C 2018, 78, 905. [CrossRef]

278. Aoki, M.; Duerr, M.; Kubo, J.; Takano, H. Multi-Component Dark Matter Systems and Their Observation Prospects. Phys. Rev. D 2012, 86, 076015. [CrossRef] 
279. Zurek, K.M. Multi-Component Dark Matter. Phys. Rev. D 2009, 79, 115002. [CrossRef]

280. Lisanti, M.; Moschella, M.; Outmezguine, N.J.; Slone, O. Testing Dark Matter and Modifications to Gravity using Local Milky Way Observables. Phys. Rev. D 2019, 100, 083009. [CrossRef]

281. McGaugh, S.S. A tale of two paradigms: The mutual incommensurability of $\Lambda$ CDM and MOND. Can. J. Phys. 2015, 93, 250. [CrossRef]

282. Sanders, R.H. Does GW170817 falsify MOND? Int. J. Mod. Phys. D 2018, 27, 14. [CrossRef]

(C) (1)

(C) 2020 by the author. Licensee MDPI, Basel, Switzerland. This article is an open access article distributed under the terms and conditions of the Creative Commons Attribution (CC BY) license (http://creativecommons.org/licenses/by/4.0/). 